\title{
Bestimmungsgründe von Arbeitszeitkonten bei Betrieben und Beschäftigten
}

\author{
Ines $\mathrm{ZAPF}^{1}$ \\ Institut für Arbeitsmarkt- und Berufsforschung (IAB)
}

\begin{abstract}
Arbeitszeitkonten ermöglichen es Arbeitszeiten zu variieren und den Arbeitseinsatz an das Arbeitsaufkommen anzupassen. Mit logistischen Regressionsmodellen und Daten des IAB-Betriebspanels wird in einem ersten Schritt analysiert, welche Faktoren Arbeitszeitkonten in Betrieben bestimmen. Die industriellen Beziehungen und betriebliche Beschäftigungsstruktur sind wesentliche Determinanten. In einem zweiten Schritt wird mit Daten des Sozio-oekonomischen Panels analysiert, welche Faktoren den Zugang von Beschäftigten zu einem Arbeitszeitkonto bestimmen. Beschäftigte mit einem höheren Maß an Humankapital haben überdurchschnittlich häufig Zugang zu einem Arbeitszeitkonto, während geringfügig beschäftigte Frauen und befristet beschäftigte Männer unterdurchschnittlich häufig Arbeitszeitkonten haben.
\end{abstract}

Keywords: Arbeitszeitflexibilität, Arbeitszeitkonten, interne Flexibilität, Work-LifeBalance, Humankapital

\section{Einleitung}

Arbeitszeitflexibilität ist in Deutschland weit verbreitet. Betriebe nutzen verschiedenste Möglichkeiten, um die Arbeitszeiten von Beschäftigten zu variieren und damit schnell auf eine veränderte ökonomische Umwelt zu reagieren. So werden Arbeitszeiten bei einer steigenden Nachfrage am Absatzmarkt vorübergehend verlängert, während temporär kürzere Arbeitszeiten bei negativen Nachfrageveränderungen die Beschäftigung stabilisieren. Mit einer Anpassung der Arbeitszeiten an das Arbeitsaufkommen können Beschäftigungsverluste vermieden oder zumindest zeitlich hinausgezögert werden. Die Möglichkeit der Beschäftigungssicherung durch interne Flexibilität ist vor dem Hintergrund der aktuellen Diskussionen um einen Fachkräftemangel besonders relevant, da Betriebe qualifizierte Beschäftigte halten wollen. Auch Beschäftigte können sich für flexible Arbeitszeiten aussprechen, um eine höhere Autonomie und Souveränität über die eigene Arbeitszeit zu erlangen und die Arbeitszeiten besser dem eigenen Lebensrhythmus anzupassen (Bosch 1996a; Teriet 1976, 1977).

Allerdings sind flexible Arbeitszeiten häufig marktgetrieben (Hildebrandt 2006), d. h. Betriebe richten die Arbeitszeiten der Beschäftigten an den Markt- und Kundenerfordernissen aus. Flexible Arbeitszeiten nach betrieblichen Bedürfnissen schränken daher die antizipierte Zeitsouveränität der Beschäftigten ein oder es entstehen Konflikte zwischen betrieblichen und

\footnotetext{
${ }^{1}$ Der Artikel basiert in Teilen auf der Dissertation von Ines Zapf (2016).
} 
individuellen Bedürfnissen (Hildebrandt 2007). Auch belegen verschiedene Studien, dass einige Merkmale flexibler Arbeitszeiten negativ auf die Gesundheit und das Privatleben von Beschäftigten wirken können. So werden Herzerkrankungen, Diabetes und Übergewicht mit Schichtarbeit assoziiert (Knutsson 2003; Paridon et al. 2012). Regelmäßige Arbeit an Abenden oder an Wochenenden geht mit einem erhöhten Risiko für arbeitsbedingte Unfälle sowie gesundheitlichen Beeinträchtigungen und einer schlechteren Work-Life-Balance einher (Arlinghaus/Nachreiner 2012). Auch eine hohe Variabilität der Arbeitszeit hat negative Auswirkungen auf die Gesundheit, das psychosoziale Wohlbefinden und den familiären- oder Freizeitbereich der Beschäftigten (Janßen/Nachreiner 2004). Dabei steigt das Risiko für Arbeitsunfälle mit Fehlzeiten, wobei die Risikoerhöhung bei Beschäftigten mit fremdbestimmten variablen Arbeitszeiten größer ist als bei Beschäftigten mit selbstbestimmten variablen Arbeitszeiten (Greubel et al. 2014).

Trotz der genannten negativen Aspekte haben flexible Arbeitszeiten eine hohe Bedeutung am deutschen Arbeitsmarkt. Diese bedeutende Rolle wurde in der jüngeren Vergangenheit intensiv im Kontext der globalen Wirtschafts- und Finanzkrise 2008/2009 diskutiert (vgl. z. B. Burda/Hunt 2011; Dietz et al. 2011; Möller 2010). Betriebe reagierten auf die Krise, indem sie die Arbeitszeiten der Beschäftigten durch eine vorübergehende Verkürzung der normalen Wochenarbeitszeit, Kurzarbeit, einem Abbau von Überstunden oder zuvor angesammelten Guthaben auf Arbeitszeitkonten zurückgefahren haben. Neben den temporären Arbeitszeitverkürzungen wurde auch die Arbeitsproduktivität verringert und somit Arbeitskräfte gehortet. Im Vergleich zu vorherigen ökonomischen Krisen wurden Arbeitszeitkonten intensiv genutzt, um Beschäftigung zu sichern. Dabei wurden die auf den Arbeitszeitkonten zuvor angesammelten Guthaben abgebaut oder sogar Zeitschulden aufgebaut (Herzog-Stein/Zapf 2014).

In der empirischen Literatur gibt es verschiedene Studien, die die Verbreitung von Arbeitszeitkonten in einem deskriptiv angelegten Rahmen untersuchen (vgl. z. B. Bauer et al. 2002; Bellmann/Gewiese 2003; Groß/Schwarz 2006). Es liegen auch vereinzelt multivariate Befunde zu den betrieblichen Bestimmungsfaktoren von Arbeitszeitkonten vor, die sich allerdings auf den Zeitraum der Wirtschafts- und Finanzkrise 2008/2009 beziehen und daher keinen längeren Zeitraum abdecken (vgl. z. B. Bellmann et al. 2012; Boeri/Brücker 2011; HerzogStein/Zapf 2014). Bislang wurde unzureichend untersucht, welche betrieblichen Bestimmungsfaktoren von Arbeitszeitkonten es gibt und ob sich diese Bestimmungsfaktoren im Zeitverlauf verändert haben. Vor dem Hintergrund der empirischen Literatur zu Arbeitszeitkonten lautet in dem vorliegenden Aufsatz die erste Forschungsfrage: Welche Faktoren determinieren Arbeitszeitkonten in Betrieben, d. h. welche Faktoren begünstigen und welche Faktoren hemmen Arbeitszeitkonten in Betrieben und gibt es Veränderungen im Zeitverlauf? Die betriebliche Entscheidung Arbeitszeitkonten einzuführen wirkt sich unmittelbar auf die Beschäftigten aus. Bislang wurde aber unzureichend analysiert, welche Beschäftigten ein Arbeitszeitkonto haben. Lediglich erste Hinweise dazu finden sich bei Bundesmann-Jansen et al. (2000) und Bauer et al. (2004), die die Verbreitung von Arbeitszeitkonten bei Beschäftigten in einem deskriptiven Rahmen untersuchen. Die zweite Forschungsfrage lautet deshalb: Welche Beschäftigten haben Zugang zu einem Arbeitszeitkonto, d. h. welche Faktoren erhöhen und welche Faktoren verringern die Wahrscheinlichkeit von Arbeitszeitkonten bei Beschäftigten? Um die beiden Forschungsfragen empirisch zu untersuchen, werden repräsentative Arbeitge- 
ber- und Arbeitnehmerbefragungen genutzt. Es werden die Betriebs- und die Individualebene berücksichtigt und zusammengeführt, um ein besseres Verständnis von Arbeitszeitkonten als betriebliches Flexibilitätsinstrument zu erhalten.

Der Aufsatz ist wie folgt gegliedert: Nach einer kurzen Definition und Beschreibung der Regulierungsmöglichkeiten werden die verschiedenen Arbeitszeitkontenmodelle vorgestellt (Abschnitt 2). Danach werden die Chancen und Vorteile, aber auch Risiken und Nachteile von Arbeitszeitkonten bei Betrieben und Beschäftigten diskutiert (Abschnitt 3). In Abschnitt 4 werden die verwendeten Daten, Variablen und die Methode vorgestellt. Abschnitt 5 stellt die deskriptiven und multivariaten Ergebnisse zu Arbeitszeitkonten im Betrieb und dem Zugang von Beschäftigten zu einem Arbeitszeitkonto dar. Der Aufsatz schließt mit einer kurzen Zusammenfassung (Abschnitt 6).

\section{Definition und Formen von Arbeitszeitkonten}

\section{Definition}

Arbeitszeitkonten gelten als modernes betriebliches Zeitbewirtschaftungsinstrument, womit eine variable Verteilung der Arbeitszeit von Beschäftigten reguliert wird (Ellguth et al. 2013; Groß/Schwarz 2008, 2010; Hildebrandt 2006; Seifert 1996, 1998). Beschäftigte sammeln auf den Arbeitszeitkonten Zeitguthaben bzw. Zeitschulden an, wenn ihre tatsächliche Arbeitszeit von ihrer vertraglich vereinbarten Arbeitszeit nach oben bzw. unten abweicht. Diese angesammelten Zeitguthaben bzw. Zeitschulden sind von den Beschäftigten innerhalb eines bestimmten Bezugszeitraums wieder auszugleichen und/oder es dürfen bestimmte Ober- bzw. Untergrenzen nicht überschritten werden (Groß/Schwarz 2008, 2010; Seifert 1998, 2001). Dabei obliegt es den Vertragsparteien, die Ober- und Untergrenzen für den Aufbau von Zeitguthaben und Zeitschulden zu vereinbaren. Ebenso ist der Ausgleichszeitraum festzulegen, innerhalb dessen das Arbeitszeitkonto wieder auszugleichen ist (Groß/Schwarz 2010; Seifert 1996, 2001, 2005). Arbeitszeitkonten bieten also die Möglichkeit, die vertraglich vereinbarte Regelarbeitszeit ungleichmäßig über einen definierten Zeitraum zu verteilen (Seifert 2014). Dabei sind Arbeitszeitkonten so konzipiert, dass sie nur die Lage und Verteilung der Arbeitszeit über einen bestimmten Bezugszeitraum ändern, während die Dauer, d. h. der Gesamtumfang der Arbeitszeit, im Beobachtungszeitraum (prinzipiell) gleich bleibt (Bauer et al. 2002; Bundesmann-Jansen et al. 2000). Im Gegensatz zu bezahlten und unbezahlten Überstunden ist durch Arbeitszeitkonten die tatsächlich geleistete Arbeitszeit im Durchschnitt nicht länger als die vertraglich vereinbarte Arbeitszeit, aber es gibt große Flexibilisierungsmöglichkeiten hinsichtlich der Lage und Verteilung. In den vergangenen Jahren wurden die Flexibilisierungsspielräume bei Arbeitszeitkonten deutlich ausgeweitet. Während im Jahr 2001 durchschnittlich 75 Plusstunden und 50 Minusstunden angesammelt werden konnten und der Ausgleichszeitraum 32 Wochen betrug, waren es im Jahr 2007 bereits 103 Plusstunden und 63 Minusstunden. Der Ausgleichszeitraum umfasste im Durchschnitt 38 Wochen (Groß 2009).

Trotz geltender tarifvertraglicher oder betrieblicher Vereinbarungen werden angesammelte Zeitguthaben von den Beschäftigten nicht immer rechtzeitig ausgeglichen oder Obergrenzen eingehalten. Prinzipiell besteht dann zunächst die Möglichkeit, dass Betriebe die ange- 
sammelten Guthaben monetär abgelten, d. h. die zusätzlich geleisteten Stunden ausbezahlen. Allerdings werden auch Zeitguthaben gekappt, wenn Obergrenzen überschritten werden. In diesem Falle leisten Beschäftigte faktisch unbezahlte Überstunden, da sie weder eine monetäre Abgeltung erhalten noch die angesammelten Guthaben in Freizeit ausgeglichen werden. Um eine monetäre Abgeltung oder Kappung von Zeitguthaben zu vermeiden, können Betriebe ihren Beschäftigten aber beispielsweise ein separates Arbeitszeitkonto mit einer längeren Ausgleichsfrist zur Verfügung stellen (Groß/Schwarz 2010). Groß/Schwarz (2006) weisen darauf hin, dass nach der Überschreitung von Obergrenzen in Betrieben (34 Prozent) in den meisten Fällen ein möglichst baldiger Freizeitausgleich erfolgen sollte (12 Prozent), die Zeitguthaben in den nächsten Ausgleichszeitraum übertragen werden (12 Prozent) oder eine monetäre Abgeltung erfolgen sollten (6 Prozent). Jedoch werden nur in wenigen Betrieben die angesammelten Zeitguthaben auf ein anderes Arbeitszeitkonto übertragen (3 Prozent), oder die Zeitguthaben verfallen in der Regel (1 Prozent). Bislang gibt es keine Erkenntnisse darüber, ob bei einem Guthabenverfall die Betriebe von Seiten der Gewerkschaften oder von Betriebs/Personalräten als Interessenvertreter der Arbeitnehmer sanktioniert werden. So dürften Beschäftigte insbesondere bei Angst vor einem Arbeitsplatzverlust oder befürchteten Karrierenachteilen nicht auf eine finanzielle Abgeltung oder einem späteren Freizeitausgleich bestehen. Vielmehr könnten die Beschäftigten die verfallenden Zeitguthaben als unbezahlte Überstunden akzeptieren.

Insgesamt gelten Arbeitszeitkonten als eine „widersprüchliche Einheit von DeRegulierung und Re-Regulierung“ (Groß/Schwarz 2010: 72). Denn auf der einen Seite lassen sich durch Arbeitszeitkonten die Dauer ${ }^{2}$, Lage und Verteilung der Arbeitszeiten von Beschäftigten variieren. Auf der anderen Seite sind Regeln zu vereinbaren und einzuhalten, die der Flexibilität einen Rahmen setzen. Beschäftigte können nur dann vor zu starken Belastungen seitens der Arbeitgeber geschützt werden, wenn die Regelungen auch eingehalten werden.

\section{Arbeitszeitkontenmodelle}

Inzwischen gibt es viele verschiedene Arbeitszeitkontenmodelle. Die Vielzahl an unterschiedlichen Modellen liegt darin begründet, dass Arbeitszeitkonten häufig nach betriebsspezifischen Bedürfnissen ausgestaltet werden. Prinzipiell lassen sich Arbeitszeitkonten aber in vier verschiedene Modelle kategorisieren. So differenziert Seifert (2001, 2005) zwischen (1) Gleitzeitkonten, (2) Überstundenkonten, (3) Bandbreiten- bzw. Korridormodellen und (4) Ansparmodellen. Diese verschiedenen Kontenmodelle lassen sich hinsichtlich der Länge des Ausgleichszeitraums in Kurzzeit- und Langzeitkonten gruppieren. Eine spezielle Form der Langzeitkonten sind die sogenannten Lebensarbeitszeitkonten (Hildebrandt 2006, 2007).

\footnotetext{
${ }^{2}$ Die Autoren beziehen die Variation der Arbeitszeitdauer auf Tage, Wochen oder Monate. Von einer Variation der Arbeitszeitdauer im eigentlichen Sinne kann allerdings nicht gesprochen werden. Denn Arbeitszeitkonten sind innerhalb eines vorgegebenen Zeitraums auszugleichen, wodurch sich die Arbeitszeitdauer innerhalb des Bezugszeitraums insgesamt nicht verändert.
} 
Abbildung 1: Modelle von Arbeitszeitkonten

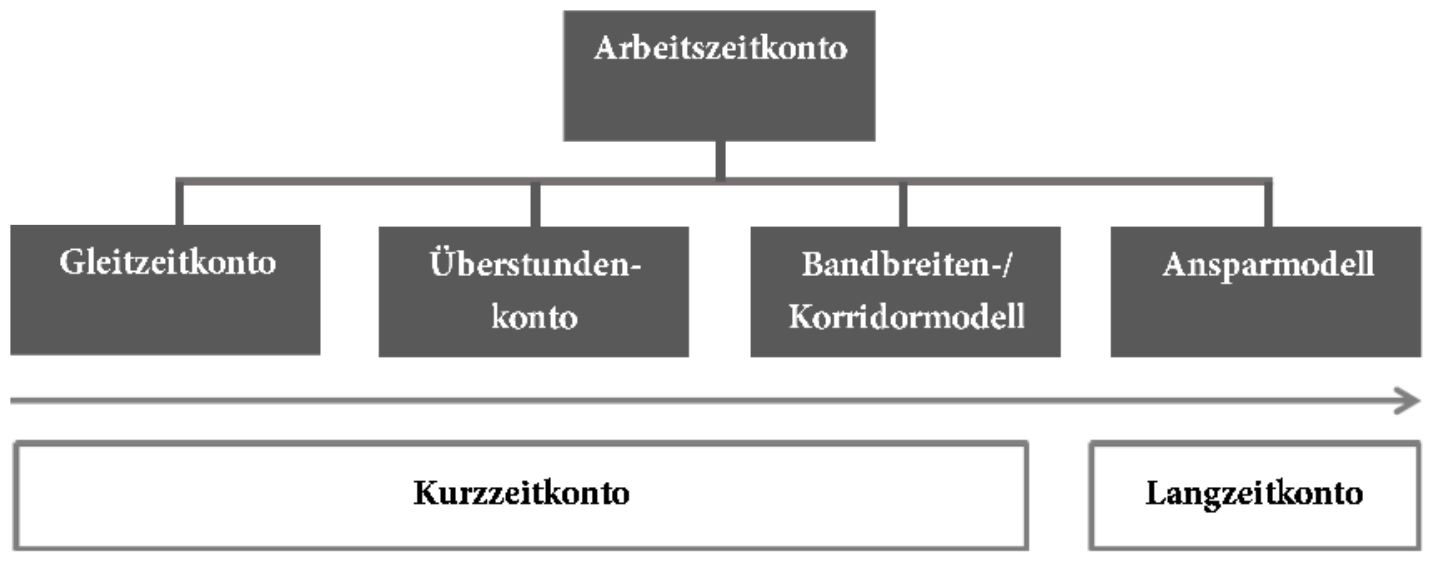

Quelle: Eigene Darstellung in Anlehnung an Seifert (1998, 2001, 2005) und Hildebrandt (2006, 2007).

In der betrieblichen Praxis vermischen sich die Modelle häufig. Teilweise haben Betriebe auch verschiedene Arbeitszeitkontenmodelle für unterschiedliche Beschäftigtengruppen. Für Beschäftigte können auch mehrere Arbeitszeitkonten nebeneinander geführt werden, wodurch Beschäftigte beispielsweise über ein Gleitzeitkonto und Ansparmodell verfügen. In diesem Fall können die angesammelten Guthaben des Gleitzeitkontos auf das Ansparkonto übertragen werden, womit die angesammelten Guthaben nicht gekappt werden und somit nicht verloren gehen (Hildebrandt 2007; Seifert 2005).

\section{Gleitzeitkonten}

Gleitzeitkonten sind die Urform von Arbeitszeitkonten. Erste Modelle sind bereits in den 1960er Jahren vor dem Hintergrund der damaligen Arbeitskräfteknappheit eingeführt worden (Seifert 2001). Durch ihre Einführung sollten die Arbeitsbedingungen verbessert werden, indem Beschäftigte vom Pünktlichkeitszwang der Normalarbeitszeit entlastet wurden. Durch einen variablen Arbeitsbeginn und ein variables Arbeitsende sollten schwierige Verkehrsverhältnisse zu den Stoßzeiten entzerrt werden (Bosch 1996a; Seifert 2005; Teriet 1976). Auch sollte die Arbeitszeitsouveränität der Beschäftigten erhöht und die Arbeitszeit besser individuellen Bedürfnissen angepasst werden können. Durch die Einführung von Gleitzeitmodellen räumten Betriebe ihren Beschäftigten nunmehr einen größeren und selbstbestimmteren Gestaltungsspielraum bei den Arbeitszeiten ein (Bosch 1996a; Bosch et al. 2005; Hinrichs/Wiesenthal 1982; Seifert 2001; Teriet 1976).

Dennoch wird die antizipierte Zeitsouveränität der Beschäftigten durch eine vergleichsweise starke betriebliche Regulierung bei den Gleitzeitkonten begrenzt. So können zwar Beginn und Ende der täglichen Arbeitszeit von den Beschäftigten frei gewählt werden, in der Regel muss aber ein bestimmter Rahmen eingehalten werden. Gleitzeitkonten zeichnen sich durch eine Kernarbeitszeit mit Anwesenheitspflicht aus. Unternehmen profitieren so von einer Mindestbesetzung zu bestimmten Tageszeiten, bei denen das Arbeitsaufkommen besonders hoch sein kann (Bauer et al. 2002; Bosch 1996a; Seifert 1998). 
Insgesamt ist bei Gleitzeitkonten lediglich ein kurzfristiger Zeitausgleich vorgesehen. Dadurch ist eine Anpassung an saisonale oder gar konjunkturelle Schwankungen kaum möglich, obwohl die Ausgleichszeiträume im Zuge immer weiter steigender betrieblicher Flexibilisierungserfordernisse länger geworden sind (Hildebrandt 2007; Seifert 1987, 1998).

\section{Überstundenkonten}

Mit Überstundenkonten können Beschäftigte lediglich Zeitguthaben aufbauen, dagegen sind Zeitschulden nicht vorgesehen. Die angesammelten Überstunden werden fakultativ oder obligatorisch durch Freizeitausgleich abgegolten. Dieser fakultative oder obligatorische Freizeitausgleich kann für alle angesammelten Überstunden oder ab einer bestimmten Stundenzahl erfolgen. Die Länge des Ausgleichszeitraums liegt häufig zwischen einem Monat und einem Jahr (Seifert 1996, 1998).

Insgesamt können durch Überstundenkonten mit einem längeren Ausgleichszeitraum saisonale Schwankungen abgefedert werden. Der betriebliche Flexibilitätsspielraum wird aber dadurch begrenzt, dass zwar Zeitguthaben aber keine Zeitschulden aufgebaut werden können. Auch gibt es bei den Regelungen zur Abgeltung der angesammelten Überstunden häufig Graubereiche. So kann von betrieblicher Seite zwar ein Freizeitausgleich vorgesehen sein. Betriebe können die mehr geleisteten Stunden aber auch ausbezahlen, sofern Beschäftigte keine Möglichkeit haben die angesammelten Überstunden abzufeiern.

\section{Bandbreiten- bzw. Korridormodelle}

Bandbreiten- oder Korridormodelle zeichnen sich durch vorab festgelegte Bandbreiten aus. Innerhalb dieser Bandbreiten kann die tatsächliche Arbeitszeit von der vertraglich vereinbarten Arbeitszeit abweichen. Die positiven oder negativen Abweichungen werden auf den Konten erfasst, die Beschäftigte innerhalb eines bestimmten Zeitraums wieder zur vereinbarten Regelarbeitszeit ausgleichen müssen. Dabei wird die tariflich oder vertraglich vereinbarte Regelarbeitszeit nur noch als ein Durchschnittswert festgelegt (Seifert 1996, 2001, 2005). Der Ausgleichszeitraum umfasst in der Regel ein Kalenderjahr.

Insgesamt bieten Bandbreiten- bzw. Korridormodelle gegenüber Gleitzeit- und Überstundenkonten eine größere Flexibilität für Betriebe und Beschäftigte. In der Regel lassen die Ober- und Untergrenzen für Zeitguthaben und Zeitschulden einen größeren Spielraum zu. Häufig ist auch der Ausgleichszeitraum länger als bei Gleitzeit- und Überstundenkonten, womit saisonale oder konjunkturelle Schwankungen abgefedert werden können.

\section{Ansparmodelle}

Mitte der 1980er Jahre wurden die ersten Ansparmodelle eingeführt, um die tariflich vereinbarten Arbeitszeitverkürzungen in Betrieben der Metall- und Elektroindustrie umzusetzen (Seifert 1996, 2005). Diese Arbeitszeitverkürzungen wurden 1984 nach einem sechswöchigen Streik beschlossen und die Wochenarbeitszeit in dieser Branche ab 1985 sukzessive verkürzt (Hinrichs 1992). Grundidee der Ansparmodelle ist, dass trotz der von den Vertragsparteien vereinbarten Arbeitszeitverkürzungen die tatsächliche wöchentliche Arbeitszeit der beschäftigten Arbeitnehmer nicht vermindert, sondern auf dem ursprünglichen Niveau gehalten 
wird. Die Differenz zwischen der tatsächlichen und der tariflich vereinbarten verkürzten Regelarbeitszeit wird auf den Konten angespart (Seifert 1996, 2001, 2005).

Bei Ansparmodellen sind - wie bei den Überstundenkonten - Zeitschulden nicht vorgesehen. Im Vergleich zu den Überstundenkonten sollen die angesammelten Zeitguthaben aber nicht kurzfristig abgebaut werden. Bei Ansparmodellen sollen die angesammelten Guthaben unter anderem für Sabbaticals, d. h. Langzeitfreistellungen im Sinne eines Sonderurlaubs während der Erwerbsphase, genutzt werden. Beschäftigte können auch beispielsweise Pflege- oder Betreuungsaufgaben in der Familie übernehmen oder Weiterbildungszeiten realisieren. Ebenfalls kann ein früherer Ausstieg aus dem Erwerbsleben erfolgen, sofern die angesammelten Guthaben erst zum Ende der Erwerbstätigkeit entnommen werden (Hildebrandt et al. 2009; Seifert 1996; Wellisch 2014).

Insgesamt sollen Ansparmodelle den Beschäftigten ermöglichen, ihre Arbeitszeit über den gesamten Lebenslauf zu variieren. Dabei stehen individuelle Bedürfnisse der Beschäftigten im Vordergrund, während betriebsspezifische Bedürfnisse in den Hintergrund treten.

Abschließend ist anzumerken, dass Gleitzeitkonten, Überstundenkonten und Bandbreiten- bzw. Korridormodelle als Kurzzeitkonten gelten (vgl. Abbildung 1). Der Ausgleichszeitraum umfasst bis zu einem Kalenderjahr, womit Betriebe kurzfristig auf Nachfrage- und Beschäftigungsschwankungen reagieren können. Gleichzeitig soll es Beschäftigten ermöglicht werden, die privaten und betrieblichen Zeitanforderungen besser miteinander abzustimmen (Bosch et al. 1992; Seifert 1996; Wolf/Beblo 2004). Hingegen gelten Ansparmodelle als Langzeitkonten, da die Ausgleichszeiträume länger als ein Jahr oder unbegrenzt sind. Die Speisung kann aus Einbringungen in Form von Zeit und Geld erfolgen. So können Beschäftigte einerseits Guthaben durch eine Arbeitszeitverlängerung aufbauen. Andererseits sehen einige Regelungen auch vor, dass Entgeltbestandteile, wie beispielsweise Urlaubsgeld oder ein festgelegter Anteil des Jahresentgeltes, in Zeitguthaben umgewandelt werden können (Bispinck 2014; Hildebrandt 2007; Seifert et al. 2013; Wotschack 2010). Wenn Beschäftigte über Langzeitkonten verfügen haben sie prinzipiell die Möglichkeit ihre Erwerbsphase neu auszugestalten. Beschäftigte erhalten die Chance Auszeiten nach individuellen Bedürfnissen während oder am Ende der Erwerbsphase zu gestalten. Langzeitkonten erlauben den Beschäftigten kontrollierte, flexible und destandardisierte Lebenslaufprofile, da sie nicht an einer betrieblichen Reaktionsfähigkeit bei Schwankungen ausgerichtet sind (Anxo/Boulin 2006; Hildebrandt 2006).

Allerdings müssen Beschäftigte auch in der Lage sein langfristig und umfangreich Guthaben aufzubauen. Bei einer langfristigen Arbeitszeitverlängerung kann es auch zu Überbelastungen kommen, die die Gesundheit der Beschäftigten gefährden. Bei einer Speisung der Langzeitkonten durch Entgelteinheiten werden Beschäftigte der oberen Einkommensgruppen privilegiert, während sich Beschäftigte mit geringen Einkommen oft keine monetäre Ansparung erlauben können (Hildebrandt 2007). Ebenfalls dürfen die angesammelten Guthaben auf Langzeitkonten nicht für betriebliche Zwecke verwendet werden. Dennoch werden in der betrieblichen Praxis die Guthaben teils auch für eine betrieblich bedingte Arbeitszeitverkürzung herangezogen, wie ein Abbau bei konjunkturellen Abschwungphasen (Esser 2007). In diesem Fall wird das Ziel einer neuen Ausgestaltung der Erwerbsphase mit flexiblen und destandardisierten Lebenslaufprofilen untergraben. 
In den Betrieben sind Langzeitkonten immer noch eine Ausnahme. So führten nach Ellguth et al. (2013) im Jahr 2010 nur 2 Prozent aller Betriebe in Deutschland Langzeitkonten. Dabei waren die angesparten Zeitguthaben in den meisten Fällen für Sabbaticals vorgesehen (35 Prozent). Auch sollten Beschäftigte Familienzeiten oder eine Verkürzung der Lebensarbeitszeit realisieren (31 Prozent bzw. 24 Prozent). Nur in wenigen Betrieben waren die angesparten Guthaben auch für Weiterbildungszeiten vorgesehen (12 Prozent).

\section{Chancen und Risiken von Arbeitszeitkonten}

Grundsätzlich sollen durch Arbeitszeitkonten sowohl betriebliche als auch individuelle Bedürfnisse der Beschäftigten realisiert werden. Arbeitszeitkonten sind aber nicht nur positiv zu bewerten, denn insbesondere für Beschäftigte können sie sich auch negativ auswirken. Im Folgenden werden mögliche Chancen und Vorteile, aber auch Risiken und Nachteile für Betriebe und Beschäftigte aufgezeigt.

\section{Chancen und Vorteile}

Durch Arbeitszeitkonten ergeben sich für Betriebe und Beschäftigte Chancen und Vorteile. Arbeitszeitkonten ermöglichen eine schnelle und flexible Anpassung der Arbeitszeiten an eine veränderte Nachfrage. Indem der Arbeitseinsatz mit dem Arbeitsaufkommen synchronisiert wird, können Betriebe auf tägliche, saisonale oder konjunkturelle Schwankungen reagieren (z. B. Bellmann/Gewiese 2003, 2004; Carstensen 1999). Dabei sind allerdings die gesetzlichen Regelungen zur Höchstarbeitszeit und die in den Tarifverträgen und Betriebsvereinbarungen ausgehandelten Regelungen zum Einsatz von Arbeitszeitkonten einzuhalten (vgl. Bispinck 1998, 2014; Esser 2007). Arbeitszeitkonten sind für Betriebe vor allem dann vorteilhaft, wenn die Nachfrageveränderungen nur vorübergehend und nicht dauerhaft sind (Hübler/Meyer 1997). Durch Arbeitszeitkonten können betriebliche Entlassungen vermindert oder vermieden werden, da zunächst die Arbeitszeit und nicht die Beschäftigtenzahl variiert wird (Bundesmann-Jansen et al. 2000; Carstensen 2000). So wird das angesammelte betriebsspezifische Humankapital der Beschäftigten im Betrieb gehalten, wodurch es langfristig zu Produktivitätssteigerungen kommen kann.

Mit Arbeitszeitkonten ergeben sich auch betriebliche Kostenvorteile. Bei positiven Nachfrageveränderungen wird eine Variation der Arbeitszeiten gegenüber der Beschäftigtenzahl bevorzugt. Betriebe vermeiden dadurch Such-, Auswahl- und Einarbeitungskosten. Entsprechend werden bei negativen Nachfrageveränderungen Entlassungskosten vermieden, wenn die Arbeitszeiten vorübergehend verkürzt werden (Bundesmann-Jansen et al. 2000; Carstensen 2000). Weitere betriebliche Kostenvorteile ergeben sich, wenn bezahlte Überstunden vermindert oder vermieden werden. Durch den Guthabenaufbau auf Arbeitszeitkonten fallen Überstundenzuschläge weg, wodurch Arbeitskosten insgesamt gesenkt werden. Dabei fallen die Transaktionskosten für Arbeitszeitkonten in der Regel geringer aus als die Kosten für Überstundenzuschläge (Carstensen 1999; Pannenberg/Wagner 2001). Variable Arbeitszeiten durch Arbeitszeitkonten vermindern für Betriebe Lagerhaltungskosten, da Arbeitszeiten besser an den Arbeitsanfall angepasst werden (Carstensen 1999; Ludewig 2001). Gleichzeitig ge- 
währleisten Arbeitszeitkonten aber eine schnelle Reaktion auf Kundenwünsche und kurze Lieferzeiten (Bellmann/Gewiese 2003, 2004).

Insgesamt lassen sich durch Arbeitszeitkonten sowohl die Arbeitsqualität steigern als auch Betriebsabläufe effizienter gestalten. Bei einem variablen Arbeitsbeginn und einem variablen Arbeitsende können Beschäftigte Arbeitszeiten wählen, in denen sie aufgrund ihres Biorhythmus produktiver sind (Hill et al. 2001). Unproduktive Arbeits- und Leerlaufzeiten werden vermieden, wenn bei einer Unterauslastung Guthaben auf Arbeitszeitkonten abgebaut und damit Arbeitszeiten vorübergehend verkürzt werden. Damit lassen sich auch Schwankungen der Stundenproduktivität dämpfen (Ludewig 2001; Wolf/Beblo 2004). Weiterhin können Arbeitszeitkonten dazu beitragen, die Attraktivität als Arbeitgeber zu steigern. Indem Betriebe ihren Beschäftigten Arbeitszeitkonten zur Verfügung stellen und diese auch für individuelle Bedürfnisse genutzt werden können, können sich Betriebe von anderen Unternehmen abheben und qualifizierte sowie motivierte Mitarbeiter leichter rekrutieren und im Betrieb halten (Goudswaard et al. 2009).

Entsprechend können auch Beschäftigte von Arbeitszeitkonten profitieren. Flexible Arbeitszeiten durch Arbeitszeitkonten können es ermöglichen Beruf und Familie besser zu vereinbaren, indem beispielsweise Arbeitszeiten mit den Öffnungszeiten von Kinderbetreuungseinrichtungen oder Schulzeiten synchronisiert werden. Beschäftigte benötigen häufig eine alltägliche Flexibilität, die bei kurzfristigen und unvorhergesehenen Ereignissen, wie Erkrankungen in der Familie, Kindergarten- oder Schulausfall, variable Arbeitszeiten ermöglichen. Ebenfalls lassen sich Beruf und familiäre Pflegeaufgaben besser miteinander vereinbaren (Hildebrandt 2006; Hill et al. 2001). Mit der Einführung von Langzeitkonten haben Beschäftigte unter anderem die Möglichkeit, Langzeitfreistellungen, Weiterbildungszeiten oder einen vorzeitigen Ruhestand zu realisieren (Hildebrandt 2007; Seifert 2014; Wotschack 2010). Insgesamt erhöht eine selbstbestimmtere Arbeitszeitgestaltung durch Arbeitszeitkonten die Zeitsouveränität der Beschäftigten, was die Zufriedenheit steigern kann und zu einer besseren Work-Life-Balance führt.

Beschäftigte erhalten durch Arbeitszeitkonten auch eine (zeitlich befristete) Beschäftigungsgarantie, da bei Nachfrageschwankungen zunächst die Arbeitszeit und nicht die Beschäftigtenzahl variiert wird (Carstensen 1999). Dabei werden Beschäftigte auch vor Entlohnungsschwankungen geschützt. Durch die Entkopplung der Löhne von der tatsächlich geleisteten Arbeitszeit schützen Arbeitszeitkonten in konjunkturellen Abschwungphasen vor geringeren Löhnen. Damit werden die Einkommen über den Konjunkturzyklus geglättet (Bellmann/Gerner 2011; Bellmann et al. 2012).

\section{Risiken und Nachteile}

Neben den genannten Chancen und Vorteilen können mit Arbeitszeitkonten auch Risiken und Nachteile für Betriebe und insbesondere für Beschäftigte einhergehen, die es zu berücksichtigen gilt. Für die Betriebe entstehen bei der Einführung und Verwaltung von Arbeitszeitkonten Kosten, wie beispielsweise Anbahnungs- und Verhandlungskosten sowie Pflege- und Dokumentationskosten (Ludewig 2001). Diese Kosten können insbesondere in kleineren Betrieben die Einführung von Arbeitszeitkonten hemmen. Werden in Betrieben Arbeitszeitkon- 
ten eingeführt, die primär nach den Interessen der Beschäftigten ausgestaltet sind, ist die betriebliche Reaktionsfähigkeit eingeschränkt. In den Betrieben entstehen Zielkonflikte zwischen den individuellen Bedürfnissen der Beschäftigten und den betrieblichen Bedürfnissen (Hildebrandt 2007). Häufig orientiert sich der Aufbau von Zeitguthaben auf Arbeitszeitkonten jedoch nach betrieblichen Erfordernissen und auch beim Abbau der Zeitguthaben haben die Betriebe Steuerungsmöglichkeiten. So ist ein beabsichtigter Freizeitausgleich häufig mit Vorgesetzten und Kollegen abzusprechen, wodurch die Zeitsouveränität der Beschäftigten eingeschränkt wird und sie nicht selbstbestimmt über ihre Arbeitszeitdauer entscheiden können (Bosch 1996a; Bosch et al. 1992; Promberger et al. 2002; Seifert 1991).

Durch die Variation der Arbeitszeiten geben Betriebe das Risiko von Nachfrageschwankungen im Absatzmarkt an die Beschäftigten weiter und wälzen einen Teil des unternehmerischen Risikos auf die Mitarbeiter ab (Bosch 1996b). Beschäftigte mit Arbeitszeitkonten können somit hohen Anforderungen und Belastungen durch den Aufbau von Guthaben ausgesetzt sein, insbesondere wenn nicht genügend Ruhe- oder Erholungsphasen zur Verfügung stehen. Bei diesen langfristig geleisteten zusätzlichen Arbeitsstunden kann die Produktivität sinken, während Sicherheits- und Gesundheitsrisiken steigen und es häufiger zu Arbeitsunfällen kommen kann (Goudswaard et al. 2009).

Leisten Beschäftigte langfristig zusätzliche Arbeitsstunden und bauen Guthaben auf Arbeitszeitkonten auf besteht das Risiko, dass diese nicht rechtzeitig ausgeglichen und Obergrenzen überschritten werden. Damit droht ein Verfall von Zeitguthaben, womit Beschäftigte faktisch unbezahlte Überstunden leisten. Arbeitszeitkonten können bei Beschäftigten auch zu Einkommensverlusten führen, wenn vormals bezahlte Überstunden durch transitorische Überstunden ersetzt werden.

Eine Einführung von Arbeitszeitkonten ist damit nicht uneingeschränkt positiv zu bewerten, da insbesondere für Beschäftigte auch Nachteile und Risiken entstehen können. Bislang fehlen aber hinreichend Daten, die eine empirische Überprüfung der hier angesprochenen Probleme ermöglichen.

\section{Daten, Variablen und Methode}

In dem vorliegenden Beitrag wird in einem ersten Schritt der Frage nachgegangen, welche Faktoren Arbeitszeitkonten in Betrieben begünstigen und welche Faktoren sie hemmen. In einem zweiten Schritt wird dann untersucht, welche Beschäftigten Zugang zu einem Arbeitszeitkonto haben. Zur Beantwortung der beiden Forschungsfragen werden zwei Datensätze mit geeigneten Variablen herangezogen. Die verwendeten Daten, Variablen sowie die zugrundeliegende Methode werden im Folgenden beschrieben.

\section{Betriebsseite}

\section{IAB-Betriebspanel}

Das IAB-Betriebspanel ist eine repräsentative Arbeitgeberbefragung zu den betrieblichen Bestimmungsgründen der Arbeitsnachfrage und wird seit 1993 in West- und seit 1996 in Ost- 
deutschland durchgeführt. Jährlich werden rund 16'000 Betriebe aller Wirtschaftszweige und Betriebsgrößen befragt. Die Interviews werden von TNS Infratest Sozialforschung GmbH durchgeführt. Die Grundgesamtheit bilden alle Betriebe mit mindestens einem sozialversicherungspflichtigem Beschäftigten.

Die Befragung liefert unter anderem Informationen zu betrieblichen Kenngrößen, wie beispielsweise Beschäftigung und Investitionen, zur Qualität der Arbeitsplätze und zum betrieblichen Qualifikationsbedarf. Weiterhin gibt es Angaben zur Lohnhöhe und zur Einbindung in das System der industriellen Beziehungen. Im mehrjährigen Abstand werden spezifische Themenblöcke in den Fragebogen integriert. Diese erfassen beispielsweise die Betriebszeiten, Überstunden und Arbeitszeitkonten. Sonderfragen zu aktuellen Schwerpunktthemen, wie beispielsweise zum Fachkräftebedarf, werden ebenfalls in die Erhebung aufgenommen (Bellmann 2002; Fischer et al. 2009).

\section{Abhängige Variable}

Die Grundlage für die abhängige Variable Arbeitszeitkonten im Betrieb bildet die folgende Frage: „Gibt es in Ihrem Betrieb/Ihrer Dienststelle Regelungen zu Arbeitszeitkonten, also von der Gleitzeit bis hin zu Jahresarbeitszeitvereinbarungen? Oder sind solche Regelungen geplant?" Die abhängige Variable ist als Dummyvariable (0/1) kodiert und nimmt den Wert 1 an, wenn Regelungen zu Arbeitszeitkonten im Betrieb vorhanden sind; die Variable nimmt den Wert 0 an, wenn Regelungen geplant oder weder vorhanden noch geplant sind.

\section{Unabhängige Variablen}

Die unabhängigen Variablen werden zu vier Hauptfaktoren gruppiert, für die ein Zusammenhang mit Arbeitszeitkonten vermutet wird. Zur ersten Gruppe der industriellen Beziehungen zählen ein geltender Tarifvertrag und ein existierender Betriebs- oder Personalrat. Die Variable Tarifbindung ist als Dummyvariable (0/1) kodiert. Sie erhält den Wert 1, wenn ein Branchentarifvertrag oder ein Haus- oder Firmentarifvertrag im Betrieb existiert; sie erhält den Wert 0, wenn es keinen Tarifvertrag gibt. Die Variable Betriebs-/Personalrat differenziert zwischen Betrieben mit einem Betriebs- oder Personalrat und Betrieben ohne Betriebs/Personalrat. Zur zweiten Gruppe der traditionellen Instrumente externer Flexibilität zählen die betrieblichen Neueinstellungen und Kündigungen, womit die Beschäftigtenzahl variiert wird. Der Anteil der Neueinstellungen und der Anteil der Kündigungen sind jeweils metrische Variablen und werden in Relation zur Gesamtbelegschaft gesetzt. Die dritte Gruppe bezieht sich auf Informationen zum Arbeitsvertrag und charakterisiert unterschiedliche Formen von Beschäftigungsverhältnissen. Dazu zählen der Anteil der Teilzeitbeschäftigten, der Anteil der geringfügig Beschäftigten, der Anteil der befristet Beschäftigten und der Anteil der Leiharbeiter im Betrieb. Diese Variablen sind metrische Variablen und beschreiben den jeweiligen Anteil an der Gesamtbelegschaft. Die vierte Gruppe beschreibt individuelle Merkmale der Beschäftigten und umfasst den Frauenanteil und den Anteil der Facharbeiter und Angestellten/Beamten mit qualifizierter Tätigkeit. Diese Variablen sind ebenfalls metrische Variablen und werden in Relation zur Gesamtbelegschaft gesetzt.

Neben den unabhängigen Variablen werden Kontrollvariablen aufgenommen, für die ein Zusammenhang mit Arbeitszeitkonten vermutet werden kann. Aufgrund eines linearen $\mathrm{Zu}-$ 
sammenhangs wird die logarithmierte Betriebsgröße, d. h. der Logarithmus der Anzahl der beschäftigten Arbeitnehmer, verwendet. Die Variable Wirtschaftsbereich differenziert zwischen 14 verschiedenen Branchen (vgl. Tabelle 3), Referenzkategorie ist das Baugewerbe. Die Variable Art des Betriebs/der Dienststelle unterscheidet zwischen eigenständigen Unternehmen bzw. einer eigenständigen Einrichtung (Referenzkategorie), einer Niederlassung/Dienststelle/Filiale, einer Zentrale/Hauptverwaltung und zwischen einer regionalen/fachlichen Mittelinstanz eines verzweigten Unternehmens oder einer verzweigten Behörde/Einrichtung. Die Rechtsform differenziert zwischen Einzelunternehmen (Referenzkategorie), einer Personengesellschaft, einer GmbH oder GmbH \& Co.KG, einer Kapitalgesellschaft, einer Körperschaft des öffentlichen Rechts/Stiftung/Anstalt/Behörde/Amt und einer sonstigen Rechtsform, wie beispielsweise Vereinen und Genossenschaften. Die Variable Technischer Stand erfasst den technischen Stand der Anlagen und der Betriebs- und Geschäftsausstattung im Vergleich zu anderen Betrieben in der Branche. Die fünfstufige Skala von (1) auf dem neuesten Stand bis (5) völlig veraltet wurde zu den drei Ausprägungen neue Technologie, weder neue noch alte Technologie (Referenzkategorie) und alte Technologie zusammengefasst. Die Variable Investitionen in EDV, Informations- und Kommunikationstechnik beschreibt, ob im vergangenen Geschäftsjahr in diesem Bereich investiert wurde. Die Region differenziert zwischen Betrieben in West- und Ostdeutschland.

\section{Beschäftigtenseite}

\section{Sozio-oekonomisches Panel}

Das Sozio-oekonomische Panel (SOEP) ist eine repräsentative Befragung von erwachsenen Personen in zufällig ausgewählten privaten Haushalten in Deutschland. Die Befragung wird seit 1984 in West- und seit 1990 in Ostdeutschland durchgeführt. Im Auftrag des Deutschen Instituts für Wirtschaftsforschung (DIW) werden jährlich rund 30'000 Personen in knapp $11^{\prime} 000$ Haushalten von TNS Infratest Sozialforschung GmbH befragt. Das SOEP liefert unter anderem Informationen zu Persönlichkeitsmerkmalen, Gesundheit, Erwerbsbeteiligung und berufliche Mobilität, Erwerbs- und Familienbiographien, Einkommensverläufen, die Zusammensetzung des Haushalts und die Wohnsituation. Wechselnde Schwerpunktthemen, wie beispielsweise zu Weiterbildung, Qualifikation und sozialer Sicherung, erweitern die Informationen (Göbel et al. 2008; Wagner et al. 2007).

\section{Abhängige Variable}

Die Grundlage für die abhängige Variable Zugang zu einem Arbeitszeitkonto bilden zwei aufeinanderfolgende Fragen: (1) „Kommt es vor, dass Sie Überstunden leisten?“ und (2) „Können diese Überstunden auch in ein sogenanntes Arbeitszeitkonto fließen, das Sie innerhalb eines Jahres oder länger mit Freizeit ausgleichen können?" Die abhängige Variable ist eine Dummyvariable (0/1) und erhält den Wert 1, wenn Überstunden in ein Arbeitszeitkonto fließen können; sie erhält den Wert 0, wenn Überstunden nicht in ein Arbeitszeitkonto fließen können.

Einschränkend ist anzumerken, dass nur befragte Personen, bei denen Überstunden vorkommen die Frage zu Arbeitszeitkonten erhalten. Um auch befragte Personen zu berücksich- 
tigen, die keine Überstunden leisten wurde folgende Annahme getroffen: Personen, die keine Überstunden leisten haben keinen Zugang zu einem Arbeitszeitkonto. Diesen Beschäftigten wird von betrieblicher Seite kein Arbeitszeitkonto zur Verfügung gestellt, da sie keine (vorübergehenden) Mehrarbeitsstunden leisten, die sie auf dem Arbeitszeitkonto verwalten können. Dennoch kann es Beschäftigte geben, die angeben, dass sie keine Überstunden leisten, aber von betrieblicher Seite werden Arbeitszeitkonten zur Verfügung gestellt. In dem Sample könnte der Anteil der Beschäftigten mit einem Arbeitszeitkonto deshalb tendenziell unterschätzt sein. Ein Vergleich der SOEP-Daten mit denen des IAB-Betriebspanels zeigt jedoch ähnliche deskriptive Ergebnisse hinsichtlich der Verbreitung von Arbeitszeitkonten bei Beschäftigten.

\section{Unabhängige Variablen}

Als unabhängige Variablen werden individuelle Faktoren und Merkmale berücksichtigt, die das Beschäftigungsverhältnis und den Arbeitsplatz der befragten Person charakterisieren. Die Variable Alter umfasst die Altersgruppen unter 30 Jahre (Referenzkategorie), 30 bis 49 Jahre sowie 50 Jahre und älter. Die Variable Kinder im Haushalt ist als Dummyvariable (0/1) kodiert. Sie erhält den Wert 1, wenn im Haushalt der befragten Person Kinder unter 16 Jahren leben; sie erhält den Wert 0, wenn dies nicht der Fall ist. Beim vertraglichen Arbeitszeitumfang wird zwischen einer Vollzeitbeschäftigung (Referenzkategorie), einer regulären Teilzeitund einer geringfügigen Beschäftigung unterschieden. Das Merkmal befristete Beschäftigung differenziert zwischen einem befristeten und einem unbefristeten Arbeitsvertrag. Bei der Leiharbeit wird zwischen Arbeitnehmern in einem Leiharbeitsverhältnis und solchen ohne Leiharbeitsverhältnis unterschieden.

Die Dauer der Betriebszugehörigkeit ist eine metrische Variable und misst, wie lange Beschäftigte bereits in einem Betrieb/einer Dienststelle tätig sind. Bei der beruflichen Position werden die benötigte Ausbildung und die Autonomie beruflichen Handelns berücksichtigt. Die benötigte Ausbildung im Beruf erfasst die Art der Ausbildung, die für die ausgeübte berufliche Tätigkeit in der Regel erforderlich ist. Hier wird zwischen kein beruflicher Ausbildungsabschluss (Referenzkategorie) und eine abgeschlossene Berufsausbildung oder ein abgeschlossenes Hochschulstudium differenziert. Letztere Kategorie umfasst sowohl ein abgeschlossenes Fachhochschulstudium als auch ein abgeschlossenes Universitäts- oder Hochschulstudium. Die Autonomie beruflichen Handelns gibt an, ob und inwieweit Beschäftigte in ihrer Arbeit selbst Entscheidungen treffen können und wie hoch der Grad der Entscheidungsfreiheit ist. Die ursprünglich fünfstufige Skala von (1) niedrige bis (5) hohe Autonomie wurde aufgrund der geringen Fallzahlen zu drei Antwortkategorien eher niedrige Autonomie (Referenzkategorie), mittlere Autonomie und eher hohe Autonomie zusammengefasst.

Neben den unabhängigen Variablen werden verschiedene Kontrollvariablen in die Modelle aufgenommen. Die berufliche Stellung differenziert zwischen Arbeitern (Referenzkategorie), Angestellten und Beamten. Die Variable Einarbeitung beschreibt die in der Regel erforderliche Art von Einarbeitung für die ausgeübte berufliche Tätigkeit und weist auf die Akquirierung von betriebsspezifischem Humankapital hin. Es wird zwischen einer kurzen Einweisung am Arbeitsplatz (Referenzkategorie), einer längeren Einarbeitung im Betrieb, und dem Besuch von Lehrgängen/Kursen, unterschieden. Die Größe des Betriebs in dem die befragte 
Person arbeitet unterscheidet zwischen Betrieben mit weniger als 20 Beschäftigten (Referenzkategorie), 20 bis 199 Beschäftigten, 200 bis 1999 Beschäftigten und über 2000 Beschäftigten. Die Variable Wirtschaftsbereich fasst die Ausprägungen nach der $\mathrm{NACE}^{3}$ in die Kategorien Produzierendes Gewerbe inklusive Baugewerbe (Referenzkategorie), Handel/Verkehr/Gastgewerbe, Sonstige Dienstleistungen und Öffentliche Dienstleistung/Erziehung/Gesundheit zusammen. Die Region differenziert zwischen West- und Ostdeutschland.

\section{Methode}

Die abhängigen Variablen wurden als dichotome, d. h. binäre Variablen spezifiziert, so dass jeweils zwischen zwei Zuständen unterschieden wird. Mit den zugrunde liegenden Daten des IAB-Betriebspanels wird zwischen den Zuständen Arbeitszeitkonten im Betrieb vs. keine Arbeitszeitkonten im Betrieb unterschieden, mit den zugrunde liegenden SOEP-Daten zwischen Zugang zu einem Arbeitszeitkonto vs. kein Zugang zu einem Arbeitszeitkonto. Die dichotome Struktur der abhängigen Variablen legt ein Logit- oder Probit-Modell als Schätzmodell nahe. Die Modelle unterscheiden sich vor allem bei den Annahmen über die Verteilung der Residuen. Logit- und Probit-Modelle führen aber häufig zu nahezu identischen Schätzergebnissen, da sich die Verteilungsfunktion der Standardnormalverteilung bei Probit-Modellen in ihrem Verlauf nur geringfügig von der Verteilungsfunktion der logistischen Regression unterscheidet.

Die empirischen Analysen wurden mit logistischen Regressionsmodellen durchgeführt. Die Basisgleichung für die Modellierung von Wahrscheinlichkeiten in der logistischen Regression lautet:

$$
\operatorname{Pr}\left(\mathrm{y}=1 \mid x_{1}, x_{2}, \ldots, x_{k}\right)=\frac{\exp \left(\beta_{0}+\beta_{1} x_{1}+\beta_{2} x_{2}+\cdots+\beta_{k} x_{k}\right)}{1+\exp \left(\beta_{0}+\beta_{1} x_{1}+\beta_{2} x_{2}+\cdots+\beta_{k} x_{k}\right)}
$$

wobei $\operatorname{Pr}\left(\mathrm{y}=1 \mid x_{1}, x_{2}, \ldots, x_{k}\right)$ die Wahrscheinlichkeit bedeutet, dass ein bestimmtes Ereignis eintritt und zwar unter dem Einfluss der unabhängigen Variablen (vgl. z. B. Greene 2008; Wooldridge 2013).

Da die $\beta$-Koeffizienten in der logistischen Regression inhaltlich nicht interpretierbar sind, werden die sogenannten Effektkoeffizienten oder Odds Ratios verwendet. Bei Odds Ratios handelt es sich um Verhältnisse von Wahrscheinlichkeitsverhältnissen. Ein Odds Ratio $<1$ gibt eine negative und ein Odds Ratio $>1$ eine positive Eintrittswahrscheinlichkeit an (Best/Wolf 2010).

In den multivariaten Analysen werden die Schätzungen getrennt für einzelne Zeitpunkte (2006, 2009, 2012) durchgeführt und diese miteinander verglichen. Die Jahre 2006 und 2012 zeichnen sich durch konjunkturelle Aufschwunphasen und das Jahr 2009 durch eine massive konjunkturelle Abschwungphase aus. Damit wird der zeitliche Trend berücksichtigt und Veränderungen im Zeitverlauf werden sichtbar. Ein Vergleich der einzelnen Zeitpunkte zeigt auf, ob Merkmale über die Zeit unterschiedlich wirken, d. h. ob Merkmale an Bedeutung gewin-

\footnotetext{
${ }^{3}$ NACE bedeutet Nomenclature Statistique des Activités Économiques dans la Communauté Européenne und beschreibt die statistische Systematik der Wirtschaftszweige in der Europäischen Gemeinschaft.
} 
nen oder verlieren. Dies zeigt sich daran, ob die Odds Ratios signifikant oder insignifikant werden. Deutliche Unterschiede deuten auf eine nichtzeitkonstante unbeobachtete Heterogenität hin. In einzelnen Jahren gibt es also Faktoren, die auf Arbeitszeitkonten wirken, die aber nicht beobachtet werden können. Das können beispielsweise Makroschocks, wie die Wirtschafts- und Finanzkrise 2008/2009, oder gesetzliche Änderungen sein. Mit getrennten Modellschätzungen für einzelne Zeitpunkte wird geprüft, ob es in einzelnen Jahren unbeobachtete Faktoren gibt. Mit diesem Vorgehen lassen sich zeitliche Entwicklungen bei den verschiedenen Determinanten darstellen, die im Rahmen von Panelanalysen nicht geleistet werden können.

Weiterhin ist anzumerken, dass die SOEP-Daten geclustert wurden. Eine Clusterung war sinnvoll, da die Adressstichproben des SOEP in einem zweistufigen Verfahren von regionalen Sampling Points und danach Haushalten gezogen werden. Die Clusterung führte dazu, dass das Pseudo- $\mathrm{R}^{2}$ nach McFadden nicht berechnet werden konnte. Stattdessen wurde das Pseudo- $\mathrm{R}^{2}$ nach McKelvey und Zavaoina's ausgegeben. Zum Vergleich wurden die logistischen Regressionen auch ohne Berücksichtigung der Clusterung durchgeführt und das Pseudo- $\mathrm{R}^{2}$ nach McFadden ausgegeben. Zwischen den Modellschätzungen zeigten sich nur sehr geringe Unterschiede, wodurch das Pseudo- $\mathrm{R}^{2}$ nach McFadden als Gütemaß herangezogen werden kann.

\section{Ergebnisse}

\section{Arbeitszeitkonten in Betrieben}

\section{Verbreitung von Arbeitszeitkonten nach Betriebsgröße}

Der Anteil der Betriebe bzw. Dienststellen mit Regelungen zu Arbeitszeitkonten ist zwischen 1999 und 2012 insgesamt gestiegen (vgl. Abbildung 2). Im Jahr 1999 gaben rund 18 Prozent der Betriebe an über Arbeitszeitkonten zu verfügen. Im Jahr 2012 waren es rund 28 Prozent. In größeren Betrieben gibt es deutlich häufiger Arbeitszeitkonten als in kleineren Betrieben. In Betrieben mit 250 und mehr Beschäftigten ist der Anteil von rund 64 auf 80 Prozent gestiegen. In Betrieben mit bis zu neun Beschäftigten stieg der Anteil von rund 12 auf 19 Prozent. 
Abbildung 2: Verbreitung von Arbeitszeitkonten nach Betriebsgröße

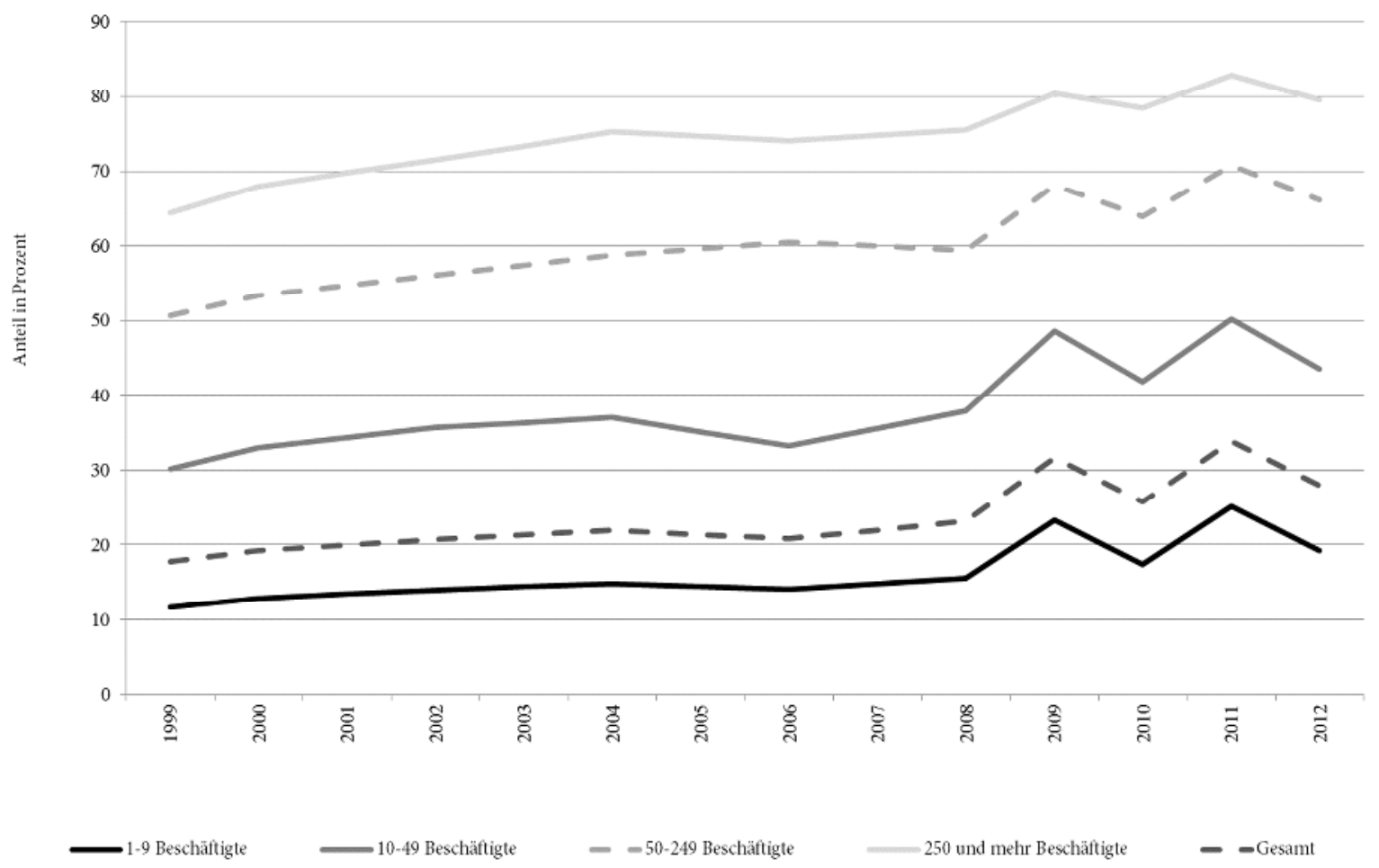

Quelle: IAB-Betriebspanel, hochgerechnete Ergebnisse, eigene Berechnungen.

Die vergleichsweise geringe Verbreitung von Arbeitszeitkonten in Kleinstbetrieben (mit bis zu neun Beschäftigten) und in Kleinbetrieben (mit bis zu 49 Beschäftigten) lässt sich mit dem verhältnismäßig großen Aufwand begründen. In kleineren Betrieben dürfte der mit einer Arbeitszeitkonteneinführung einhergehende Aufwand höher sein als der daraus entstehende Nutzen einer formalen Flexibilisierung. In kleineren Betrieben scheint es eher eine informelle Praxis zu einer flexiblen Arbeitszeitgestaltung zu geben, die eine formale Regelung durch Arbeitszeitkonten überflüssig erscheinen lässt (Bellmann/Gewiese 2003; Bosch 1996a; Kraetsch/Trinczek 1998). Der hohe Anteil der Arbeitszeitkontenbetriebe unter den Großbetrieben (mit mindestens 250 Beschäftigten) ist auf den Fixkostencharakter von Arbeitszeitkonten zurückzuführen. Bei der Arbeitszeitkonteneinführung fallen unterschiedliche Kosten an, wie Anbahnungs- und Verhandlungskosten sowie Kosten für die Pflege und Dokumentation. Aufgrund der Fixkostendegression sinken die durchschnittlichen Kosten von Arbeitszeitkonten mit steigender Betriebsgröße, wodurch sie für Großbetriebe günstiger sind (Ludewig 2001).

Auffällig ist die hohe Volatilität des Anteils der Arbeitszeitkontenbetriebe im Zeitraum von 2008 bis 2012. Während der Anteil über alle Betriebsgrößenklassen im Jahr 2009 sprunghaft angestiegen ist (von 23,2 Prozent auf 31,6 Prozent), ist er im Jahr 2010 wieder deutlich zurückgegangen (25,8 Prozent). Ein Jahr später ist er wieder erneut angestiegen (33,8 Prozent) und im Jahr 2012 gesunken (28,0 Prozent). In kleineren Betrieben sind die Schwankungen noch stärker ausgeprägt als in größeren Betrieben. Die stärkeren Schwankungen in kleineren 
Betrieben können darauf zurückgeführt werden, dass kleinere Betriebe häufig kein computergestütztes und hochautomatisiertes System im Sinne eines Arbeitszeitkontos haben, sondern eher einfachere Formen nutzen, um Arbeitszeitveränderungen zu dokumentieren, z. B. (handschriftliche) Eintragungen von Beschäftigten. Während der Wirtschafts- und Finanzkrise wurden vermutlich vor allem in kleineren Betrieben Arbeitszeitkonten als relativ einfaches und wenig technologisch entwickeltes Dokumentationssystem auch kurzfristig eingeführt und danach wieder aufgegeben bzw. nicht mehr genutzt. Dagegen verfügen größere Betriebe eher über technologisch hoch entwickelte und computergestützte Systeme. Diese werden nach ihrer Einführung nicht ohne höhere Kosten wieder abgeschafft, wodurch der Anteil der Arbeitszeitkontenbetriebe weniger stark schwankt. Die bei allen Betriebsgrößenklassen auftretenden Schwankungen können jedoch auch auf einen Messfehler hindeuten. ${ }^{4}$

\section{Verbreitung von Arbeitszeitkonten nach Branche}

Entsprechend der Gesamtentwicklung ist der Anteil der Arbeitszeitkontenbetriebe in den meisten Branchen zwischen 1999 und 2012 gestiegen (vgl. Tabelle 1). Im sekundären Sektor gibt es die deutlichste Zunahme in der Produktionsgüterindustrie und Grundstoffverarbeitung (+16,8 Prozentpunkte) sowie in der Investitions- und Gebrauchsgüterindustrie $(+15,4$ Prozentpunkte). Im tertiären Sektor ist der Anstieg bei den Organisationen ohne Erwerbscharakter, Öffentliche Verwaltung und Sozialversicherung sowie in der Branche Erziehung und Unterricht am stärksten (+17,0 bzw. +15,8 Prozentpunkte). Gründe für die deutlichen $\mathrm{Zu}$ nahmen insbesondere im tertiären Sektor dürften einerseits eine intendierte Steigerung der Arbeitgeberattraktivität und andererseits der auch hier zunehmende Kostendruck sein, der eine Einführung von Arbeitszeitkonten begünstigt. Entgegen der Gesamtentwicklung nimmt im Bereich Bergbau, Energie- und Wasserversorgung sowie im Kredit- und Versicherungsgewerbe der Anteil der Arbeitszeitkontenbetriebe ab (-5,1 bzw. -7,6 Prozentpunkte).

\footnotetext{
${ }^{4}$ Im Zeitraum von 2008 bis 2012 wurde weder die Erhebungsmethode noch die Frage zu den Arbeitszeitkonten verändert. Da in 2009 und 2011 die vorangegangene Frage zu geleisteten Überstunden keine Informationen zur Abgeltung beinhaltete, während dies in 2008, 2010 und 2012 der Fall war, könnte diese Frage auf die nachfolgende Frage zu den Arbeitszeitkonten ausgestrahlt haben. So kann angenommen werden, dass Betriebe, die normalerweise einen Freizeitausgleich von Überstunden ankreuzen in 2009 und 2011 die Regelungen zu Arbeitszeitkonten angekreuzt haben, wodurch der Anteil der Arbeitszeitkontenbetriebe jeweils deutlich steigt. Diese Ausstrahlung auf andere Fragen wird auch als Fragereiheneffekt oder „Halo-Effekt“ bezeichnet (Schief 2003).
} 
Tabelle 1: Verbreitung von Arbeitszeitkonten nach Branche

\begin{tabular}{lccccccccc} 
& $\mathbf{1 9 9 9}$ & $\mathbf{2 0 0 2}$ & $\mathbf{2 0 0 4}$ & $\mathbf{2 0 0 6}$ & $\mathbf{2 0 0 8}$ & $\mathbf{2 0 0 9}$ & $\mathbf{2 0 1 0}$ & $\mathbf{2 0 1 1}$ & $\mathbf{2 0 1 2}$ \\
\hline Land- und Forstwirstschaft/Fischerei & 22.0 & 26.8 & 27.0 & 16.9 & 26.6 & 35.5 & 23.9 & 34.5 & 27.7 \\
Bergbau/Energie- und Wasserversorgung & 58.6 & 53.6 & 51.1 & 59.4 & 51.7 & 56.3 & 50.6 & 55.3 & 53.4 \\
Verbrauchsgüterindustrie/Nahrungs- und Genussmittel & 15.5 & 20.0 & 21.8 & 22.8 & 25.6 & 30.8 & 27.7 & 31.5 & 28.9 \\
Produktionsgüterindustrie/Grundstoffverarbeitung & 27.8 & 33.5 & 44.2 & 40.9 & 41.7 & 49.7 & 51.0 & 52.8 & 44.6 \\
Investitions- und Gebrauchsgüterindustrie & 26.8 & 37.4 & 37.9 & 34.6 & 41.6 & 49.2 & 42.7 & 47.8 & 42.2 \\
Baugewerbe & 24.1 & 26.5 & 27.2 & 28.0 & 29.9 & 35.7 & 29.5 & 40.9 & 32.0 \\
Handel/Reparatur & 13.1 & 16.6 & 16.6 & 17.9 & 19.7 & 28.0 & 23.1 & 30.4 & 24.7 \\
Verkehr/Information und Kommunikation & 12.9 & 26.6 & 23.5 & 18.9 & 17.3 & 32.6 & 27.3 & 36.2 & 27.0 \\
Kredit/Versicherungen & 32.0 & 30.1 & 27.8 & 20.1 & 25.7 & 27.8 & 26.2 & 28.3 & 24.4 \\
Gastgewerbe & 8.9 & 9.0 & 14.0 & 10.3 & 10.7 & 21.2 & 13.0 & 22.2 & 16.4 \\
Erziehung und Unterricht & 14.9 & 20.2 & 23.3 & 20.8 & 24.4 & 32.7 & 34.0 & 34.6 & 30.7 \\
Gesundheits-/Sozial- und Veterinärwesen & 15.0 & 16.7 & 16.9 & 20.0 & 22.6 & 32.4 & 24.4 & 35.2 & 28.5 \\
Sonstige Dienstleistungen & 19.3 & 17.7 & 20.2 & 17.4 & 19.7 & 28.0 & 21.0 & 28.5 & 25.5 \\
Organisationen ohne Erwerbscharakter/Öffentliche Verwal- & & & & & & & & & \\
tung/Sozialversicherung & & & & & & & & \\
\hline Gesamt & 24.2 & 25.5 & 29.6 & 32.7 & 32.6 & 40.9 & 40.3 & 52.1 & 41.2 \\
\hline
\end{tabular}

Quelle: IAB-Betriebspanel, hochgerechnete Ergebnisse, eigene Berechnungen.

Während der Wirtschafts- und Finanzkrise 2008/2009 ist der Anteil der Arbeitszeitkontenbetriebe am stärksten in den Branchen Verkehr, Information und Kommunikation sowie im Gastgewerbe gestiegen (+15,3 bzw. 10,5 Prozentpunkte). In den von der Krise stark getroffenen Branchen des sekundären Sektors zeigen sich zwar ebenfalls Zunahmen, allerdings sind diese nicht so stark ausgeprägt wie in den meisten Branchen des tertiären Sektors. Dies könnte darauf zurückzuführen sein, dass Arbeitszeitkonten im sekundären Sektor bereits weit verbreitet sind und daher die Zuwächse im Krisenzeitraum geringer waren als im tertiären Sektor.

\section{Nutzungsintensität in Arbeitszeitkontenbetrieben}

Die Nutzungsintensität gibt an, wie viel Prozent der beschäftigten Arbeitnehmer über ein Arbeitszeitkonto verfügen, gegeben dass diese in den Betrieben bzw. Dienststellen existieren.

Insgesamt betrachtet ist die Nutzungsintensität zwischen 1999 und 2012 von rund 83 auf 89 Prozent gestiegen (vgl. Tabelle 2). Bei einer Differenzierung nach der Betriebsgröße zeigt sich, dass in kleineren Betrieben mit Arbeitszeitkonten anteilig noch mehr Beschäftigte über ein Arbeitszeitkonto verfügen, d. h. dazu Zugang haben, als in größeren Betrieben. Im Jahr 2012 hatten rund 90 Prozent der Mitarbeiter in Kleinstbetrieben mit Arbeitszeitkonten auch Zugang zu Arbeitszeitkonten und in Großbetrieben waren es rund 84 Prozent. Die höhere Nutzungsintensität in kleineren Betrieben ist jedoch plausibel, da Arbeitszeitkonten in kleineren Betrieben in Relation zur Beschäftigtenzahl höhere Kosten erzeugen als in größeren Betrieben. Daher erscheinen Arbeitszeitkonten in kleineren Betrieben nur dann sinnvoll, wenn diese allen Beschäftigten zur Verfügung stehen. Ebenfalls ist die betriebliche Arbeitszeitpolitik 
in kleineren Betrieben häufig nicht differenziert, sondern die Arbeitszeitregelungen gelten uneingeschränkt für alle Beschäftigte. Dagegen ist in größeren Betrieben die Arbeitszeitpolitik häufig differenzierter und bei unterschiedlichen Beschäftigtengruppen werden verschiedene Instrumente herangezogen, um Arbeitszeiten zu flexibilisieren. Damit können Arbeitszeitkonten nur einem bestimmten Teil der beschäftigten Arbeitnehmer zur Verfügung stehen, während bei anderen Beschäftigtengruppen beispielsweise auf bezahlte Überstunden zurückgegriffen wird oder Vertrauensarbeitszeit ohne eine dokumentierte Arbeitszeit weiter verbreitet ist.

Bei einer Differenzierung nach Branchen zeigt sich, dass die Nutzungsintensität in der Produktionsgüterindustrie und Grundstoffverarbeitung, im Gastgewerbe und bei den sonstigen Dienstleistungen zurückgegangen ist. Bei allen anderen Branchen zeigt sich ein Anstieg. Überdurchschnittlich hoch war die Nutzungsintensität im Jahr 2012 im Gesundheits-, Veterinär- und Sozialwesen und in der Branche Kredit und Versicherungen. Im Gastgewerbe und in der Produktionsgüterindustrie und Grundstoffverarbeitung war die Nutzungsintensität am geringsten.

Tabelle 2: Nutzungsintensität von Arbeitszeitkonten

\begin{tabular}{|c|c|c|}
\hline & 1999 & 2012 \\
\hline \multicolumn{3}{|l|}{ Betriebsgröße } \\
\hline 1-9 Beschäftigte & 85.4 & 90.1 \\
\hline 10-49 Beschäftigte & 82.3 & 87.7 \\
\hline 50-249 Beschäftigte & 78.5 & 84.9 \\
\hline 250 und mehr Beschäftigte & 73.2 & 84.4 \\
\hline \multicolumn{3}{|l|}{ Branche } \\
\hline Land- und Forstwirstschaft/Fischerei & 61.9 & 88.1 \\
\hline Bergbau/Energie- und Wasserversorgung & 82.2 & 88.5 \\
\hline Verbrauchsgüterindustrie/Nahrungs- und Genussmittel & 73.8 & 83.9 \\
\hline Produktionsgüterindustrie/Grundstoffverarbeitung & 86.6 & 83.0 \\
\hline Investitions- und Gebrauchsgüterindustrie & 85.1 & 89.0 \\
\hline Baugewerbe & 82.6 & 87.3 \\
\hline Handel/Reparatur & 76.7 & 87.3 \\
\hline Verkehr/Information und Kommunikation & 86.4 & 90.6 \\
\hline Kredit/Versicherungen & 93.4 & 91.2 \\
\hline Gastgewerbe & 85.5 & 82.7 \\
\hline Erziehung und Unterricht & 85.4 & 87.4 \\
\hline Gesundheits-/Sozial- und Veterinärwesen & 81.0 & 91.3 \\
\hline Sonstige Dienstleistungen & 93.2 & 89.6 \\
\hline Organisationen ohne Erwerbscharakter/Öffentliche Verwaltung/Sozialversicherung & 82.6 & 89.7 \\
\hline Gesamt & 83.1 & 88.5 \\
\hline
\end{tabular}

Quelle: IAB-Betriebspanel, hochgerechnete Ergebnisse, eigene Berechnungen.

\section{Ergebnisse der multivariaten Analyse}

Bei den Determinanten von Arbeitszeitkonten in einem Betrieb lassen sich folgende Ergebnisse festhalten: Erstens zeigt sich ein positiver Zusammenhang zwischen den industriellen Be- 
ziehungen und Arbeitszeitkonten (vgl. Tabelle 3). Tarifgebundene Betriebe und Betriebe mit Betriebs- oder Personalrat haben im Vergleich zu nicht tarifgebundenen Betrieben und Betrieben ohne Betriebs-/Personalrat eine höhere Wahrscheinlichkeit von Arbeitszeitkonten. So erhöht sich im Jahr 2012 das Risiko bzw. die Chance von Arbeitszeitkonten für tarifgebundene Betriebe signifikant um rund 31 Prozent im Vergleich zu nicht tarifgebundenen Betrieben. Für Betriebe mit Betriebs-/Personalrat erhöht sich das Risiko bzw. die Chance von Arbeitszeitkonten um rund 63 Prozent. Die Ergebnisse weisen darauf hin, dass eine Tarifbindung und ein existierender Betriebs-/Personalrat wichtige Bestimmungsfaktoren für Arbeitszeitkonten sind. In den vergangenen Jahrzehnten hat aber eine Kompetenzverschiebung von der tariflichen auf die betriebliche Ebene stattgefunden. ${ }^{5}$ Die Arbeitszeiten werden im Rahmen von Betriebsvereinbarungen mehr und mehr dezentral geregelt (vgl. z. B. Berg 2008). So werden in Tarifverträgen oftmals nur die Rahmenbedingungen für Arbeitszeitkonten festgelegt, die konkrete Regulierung und Ausgestaltung findet dann auf der betrieblichen Ebene statt. Durch das Mitbestimmungsrecht der Betriebs-/Personalräte bei Arbeitszeitkonten können auch die Wünsche der Beschäftigten besser berücksichtigt werden. Betriebs-/Personalräte sprechen sich für Arbeitszeitkonten aus, wenn eine größere Arbeitszeitautonomie für die Beschäftigten erwartet wird (Ellguth/Promberger 2004). Auch handeln Betriebs-/Personalräte häufig im Sinne der Gewerkschaften und akzeptieren den Kompromiss einer höheren Arbeitszeitflexibilität im Tausch für Beschäftigungssicherheit (Ludewig 2001).

Zweitens zeigt sich nur teilweise ein Zusammenhang zwischen den traditionellen Instrumenten externer Flexibilität und Arbeitszeitkonten als Instrument interner Flexibilität. Tendenziell steigt mit einem zunehmenden Anteil an Neueinstellungen auch die Wahrscheinlichkeit von Arbeitszeitkonten. Die Effektkoeffizienten sind aber nur 2009 und 2012 signifikant. Dieser Befund weist gegen Ende des Beobachtungszeitraums eher auf ein komplementäres Verhältnis von Neueinstellungen und Arbeitszeitkonten hin. Da der Aufbau von Zeitguthaben auf Arbeitszeitkonten infolge der gesetzlichen Regelungen zur täglichen Höchstarbeitszeit begrenzt ist und auch tarifvertragliche oder betriebliche Vereinbarungen Obergrenzen für Zeitguthaben schaffen, stoßen Arbeitszeitkonten bei der Variation des Arbeitseinsatzes an Grenzen. So werden auch in Arbeitszeitkontenbetrieben Neueinstellungen notwendig, um den Arbeitseinsatz langfristig an ein höheres Arbeitsaufkommen anzupassen. Dagegen gibt es keinen signifikanten Zusammenhang zwischen betriebsbedingten Kündigungen und Arbeitszeitkonten. Damit scheinen betriebsbedingte Kündigungen Arbeitszeitkonten nicht grundsätzlich im Wege zu stehen und eine Einführung und Nutzung zu erschweren. Dies erscheint insbesondere dann plausibel, wenn betriebsbedingte Kündigungen erst ausgesprochen werden, wenn andere Maßnahmen bereits weitgehend ausgeschöpft wurden und Betriebe keine anderen Möglichkeiten mehr sehen, um den Arbeitseinsatz an das Arbeitsaufkommen anzupassen.

Drittens weisen die Ergebnisse überwiegend auf Zusammenhänge zwischen den Merkmalen des Arbeitsvertrags und Arbeitszeitkonten hin. Mit einem zunehmenden Anteil von Teilzeit- und geringfügig Beschäftigten reduziert sich die Wahrscheinlichkeit von Arbeitszeitkon-

\footnotetext{
${ }^{5}$ Diese Kompetenzverschiebung resultiert aus den zunehmenden betrieblichen Öffnungsklauseln mit denen die variierende Arbeitszeitverteilung bei tariflich definierten Ausgleichszeiträumen organisiert werden kann.
} 
ten. Der Teilzeitanteil ist aber nur 2006, der Anteil geringfügig Beschäftigter in den Jahren 2009 und 2012 signifikant. Im Beobachtungszeitraum scheint sich also der negative Zusammenhang von der regulären Teilzeitbeschäftigung hin zur geringfügigen Beschäftigung verschoben zu haben. Geringfügige Beschäftigungen schaffen für Betriebe ebenfalls eine Möglichkeit von zeitlicher Flexibilität. Durch geringfügige Beschäftigungsverhältnisse kann ein zusätzlicher Arbeitskräftebedarf außerhalb der Kernarbeitszeiten oder am Wochenende abgedeckt werden. Seit dem Jahr 2003 ist die Anzahl geringfügiger Beschäftigungsverhältnisse deutlich gestiegen, da die Geringfügigkeitsgrenze auf 400 Euro angehoben und damit die betriebliche Nutzung erleichtert wurde. ${ }^{6}$ Mit einem steigenden Anteil geringfügig Beschäftigter kann das betriebliche Interesse an Arbeitszeitkonten aus zwei Gründen sinken: Zum einen ist das Potential dieser Beschäftigtengruppe für den Aufbau von Zeitguthaben im Zuge der Verdienstgrenze gering. Zum anderen besteht mit einem variierenden Anteil geringfügig Beschäftigter bis zu einem gewissen Maße eine Substitutionsmöglichkeit. Betriebe, die durch geringfügige Beschäftigung flexibel sind, können sich deshalb gegen Arbeitszeitkonten aussprechen.

Bei den Merkmalen des Arbeitsvertrags zeigt sich ein negativer Zusammenhang zwischen dem Befristungsanteil und Arbeitszeitkonten. In diesem Kontext sprechen die Ergebnisse für einen gewissen Trade-off zwischen Maßnahmen interner und externer Flexibilität. D. h. mit einer zunehmenden Befristungsquote als vergleichsweise neues Instrument externer Flexibilität reduziert sich die Wahrscheinlichkeit von Arbeitszeitkonten als Instrument interner Flexibilität. Bei einer höheren Befristungsquote scheint es für Betriebe durchaus schwieriger zu sein, Arbeitszeitkonten effektiv zu gestalten und damit den Arbeitseinsatz zu variieren. Einerseits kann dies darauf zurückzuführen sein, dass Beschäftigte mit einem relativ kurzen und zeitlich begrenztem Beschäftigungsverhältnis weniger in der Lage sind Zeitguthaben oder Zeitschulden nach betrieblichen Bedürfnissen aufzubauen. Andererseits werden externe Flexibilitätsinstrumente bei einer relativ zur Stammbelegschaft steigenden Randbelegschaft bedeutender. Der positive Zusammenhang zwischen dem Leiharbeiteranteil und Arbeitszeitkonten spricht für die These, dass Betriebe die Leiharbeit komplementär zu Arbeitszeitkonten nutzen, um intern und extern flexibel zu sein. Hierbei dürfte es sich vor allem um Betriebe mit hohem Flexibilitätsbedarf handeln. Die Leiharbeit kann dabei auch ein Puffer für die Stammbelegschaft darstellen, indem bei einem Nachfragerückgang der Umfang der Leiharbeit reduziert wird und (Stamm-)Beschäftigte Guthaben auf Arbeitszeitkonten abbauen. In solchen Betrieben findet eine zunehmende Polarisierung zwischen gut geschützten Insidern und prekär Beschäftigten an den Rändern der Belegschaft statt.

Viertens weisen die Ergebnisse auf verschiedene Zusammenhänge zwischen individuellen Beschäftigtenmerkmalen und Arbeitszeitkonten hin. Arbeitszeitkonten scheinen tendenziell unterdurchschnittlich häufig in Betrieben mit einem höheren Frauenanteil vorzukommen. Allerdings ist der Effektkoeffizient im Jahr 2006 nur schwach und im Krisenjahr 2009 nicht signifikant. Dieses Ergebnis lässt tendenziell vermuten, dass es für Betriebe mit einer höheren Frauenquote schwieriger erscheint Arbeitszeitkonten zur Arbeitszeitflexibilisierung nach betrieblichen Bedürfnissen zu nutzen. Frauen benötigen eher flexible Arbeitszeiten nach individuellen Bedürfnissen, um Beruf und Familie zu vereinbaren. Dagegen können die Arbeitszei-

\footnotetext{
${ }^{6}$ Zum 1.1.2013 wurde die Verdienstgrenze um weitere 50 Euro auf 450 Euro angehoben.
} 
ten von Männern eher an die betriebliche Situation angepasst werden, da sie in geringerem Maße familiäre Verpflichtungen haben, die es mit dem Beruf zu vereinbaren gilt.

Der positive Zusammenhang zwischen dem Anteil der Facharbeiter sowie qualifizierten Beschäftigten und Arbeitszeitkonten weist darauf hin, dass Betriebe bei diesen Beschäftigtengruppen an langfristigen Arbeitsverhältnissen interessiert sind. Facharbeiter und qualifizierte Beschäftigte haben in höherem Maße allgemeines und betriebsspezifisches Humankapital angesammelt. Je höher ihr Anteil an der Gesamtbelegschaft ist, desto sinnvoller sind Maßnahmen der internen Flexibilität, um das angesammelte Humankapital im Betrieb zu halten und zu nutzen. Daher wird bei negativen Nachfrageveränderungen zunächst versucht, den Arbeitseinsatz durch einen Guthabenabbau auf Arbeitszeitkonten nach unten anzupassen und betriebsbedingte Kündigungen zu vermeiden. Bei positiven Auftragsveränderungen kann das Humankapital dieser Beschäftigtengruppe noch mehr genutzt werden, indem die Arbeitszeit durch den Guthabenaufbau vorübergehend verlängert wird.

Die deskriptiven Befunde haben gezeigt, dass Arbeitszeitkonten in größeren Betrieben und in den Branchen des sekundären Sektors weiter verbreitet sind als in kleineren Betrieben und in den Branchen des tertiären Sektors. In den multivariaten Analysen können diese Befunde bestätigt werden. Mit zunehmender Betriebsgröße steigt die Wahrscheinlichkeit von Arbeitszeitkonten. Größere Betriebe nutzen überdurchschnittlich häufig Arbeitszeitkonten als modernes Instrument zur Arbeitszeitflexibilisierung. Im Vergleich zum Baugewerbe finden sich Arbeitszeitkonten unterdurchschnittlich häufig in den meisten Branchen des tertiären Sektors, wie im Bereich Handel und Reparatur, Verkehr/Information und Kommunikation sowie Erziehung und Unterricht. Die immer noch stärkere Verbreitung von Arbeitszeitkonten im sekundären Sektor lässt sich vor allem darauf zurückführen, dass der Kompromiss einer höheren Arbeitszeitflexibilität im Austausch für Arbeitszeitverkürzung und Beschäftigungssicherheit zuerst im sekundären Sektor umgesetzt wurde. Daraus sind Arbeitszeitkonten als betriebliches Flexibilitätsinstrument hervorgegangen, um die Arbeitszeit nicht mehr nur durch klassische Instrumente, wie bezahlte und unbezahlte Überstunden, zu variieren.

Die Art des Betriebs bzw. der Dienststelle scheint für Arbeitszeitkonten nur von geringer Bedeutung zu sein. Im Jahr 2012 erhöht sich das Risiko bzw. die Chance von Arbeitszeitkonten für Zentralen oder Hauptverwaltungen signifikant um rund 47 Prozent. Bei den anderen Betriebsarten zeigen sich keine signifikanten Unterschiede zum eigenständigen Unternehmen. Betriebe mit Rechtsform Personengesellschaft, GmbH bzw. GmbH \& Co. KG, Kapitalgesellschaft und Körperschaft des öffentlichen Rechts/Stiftung/Anstalt/Behörde und Amt setzen im Vergleich zu Einzelunternehmen überdurchschnittlich häufig Arbeitszeitkonten ein. 
Tabelle 3: Determinanten von Arbeitszeitkonten in Betrieben

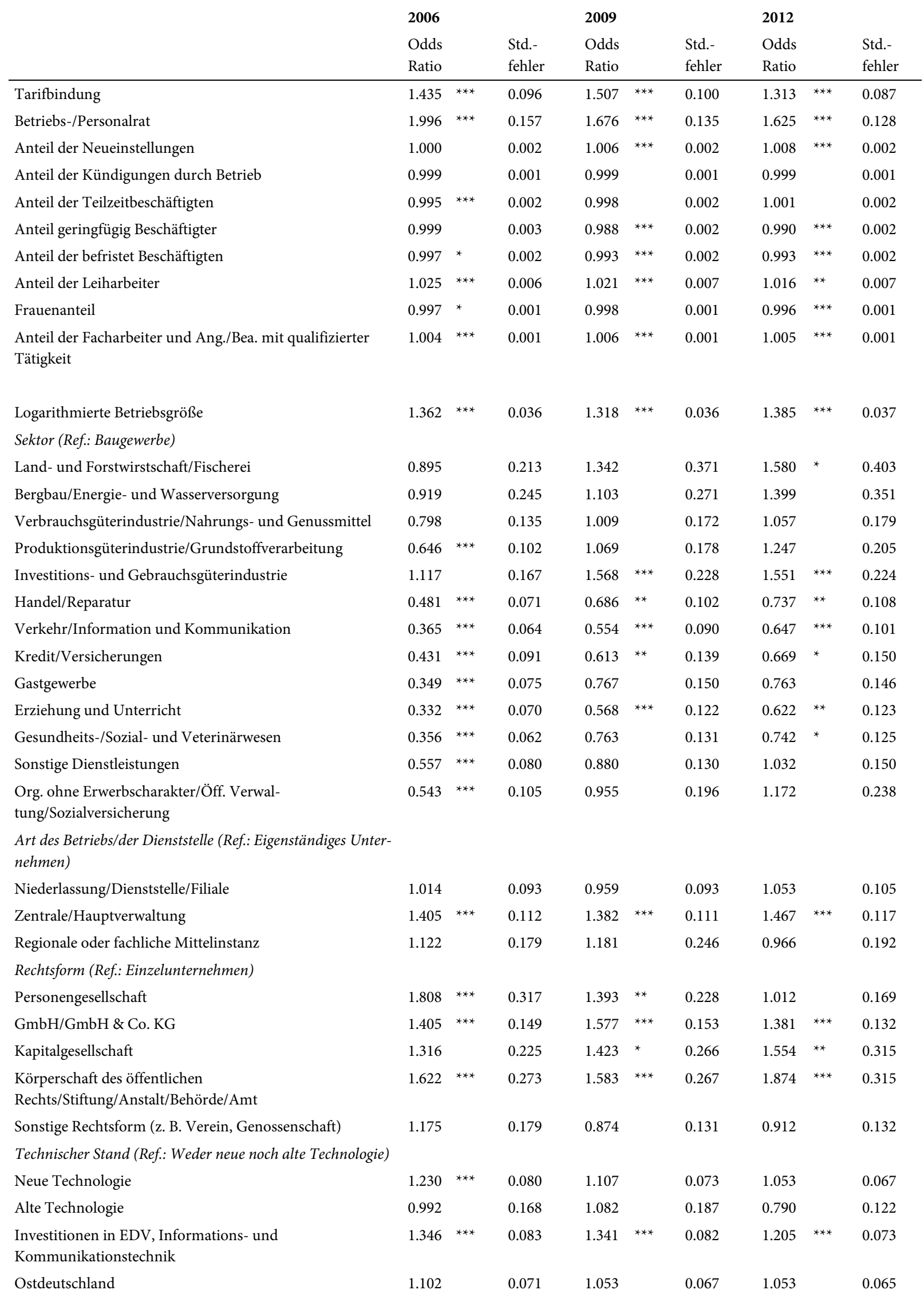




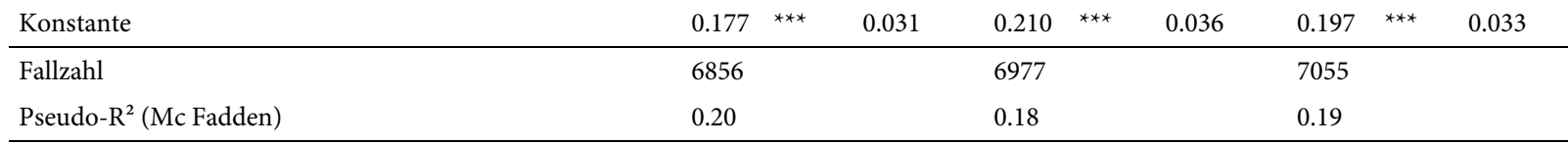

${ }^{*} \mathrm{p}<0.1,{ }^{* *} \mathrm{p}<0.05,{ }^{* * *} \mathrm{p}<0.01$

Quelle: IAB-Betriebspanel, eigene Berechnungen.

Zwischen dem technischen Stand der Anlagen und Arbeitszeitkonten gibt es keinen signifikanten Zusammenhang, hingegen zeigt sich ein signifikant positiver Zusammenhang bei den getätigten Investitionen im Bereich EDV, Informations- und Kommunikationstechnik. Bei beiden Merkmalen hätte man einen positiven Zusammenhang vermuten können, da ein moderner technischer Stand der Anlagen und getätigte Investitionen im Bereich EDV, Informations- und Kommunikationstechnik eine gute Basis für ein modernes Arbeitszeiterfassungssystem darstellen (Bellmann/Gewiese 2004). Die Ergebnisse zeigen keine signifikanten Unterschiede zwischen ost- und westdeutschen Betrieben in Hinblick auf Arbeitszeitkonten.

\section{Arbeitszeitkonten bei Beschäftigten}

\section{Verbreitung von Arbeitszeitkonten nach Arbeitszeitumfang und Geschlecht}

Bei der Verbreitung von Arbeitszeitkonten nach Geschlecht zeigt sich, dass anteilig etwas mehr Männer Zugang zu einem Arbeitszeitkonto haben als Frauen. Allerdings ist der Unterschied nur im Jahr 2002 auch signifikant. Im Jahr 2002 hatten rund 43 Prozent der Männer und 39 Prozent der Frauen Zugang zu einem Arbeitszeitkonto, im Jahr 2012 waren es rund 54 bzw. 53 Prozent. Zu ähnlichen Ergebnissen kommen Bauer et al. (2004) im Rahmen einer Beschäftigtenbefragung. Danach hatten im Jahr 2003 rund 45 Prozent der Männer und 37 Prozent der Frauen ein Arbeitszeitkonto. Bei einer weiteren Differenzierung nach dem vertraglichen Arbeitszeitumfang weisen die deskriptiven Befunde darauf hin, dass vollzeitbeschäftigte Männer und Frauen häufiger Zugang zu einem Arbeitszeitkonto haben als teilzeitbeschäftigte Männer und Frauen (vgl. Tabelle 4). Bei Frauen sind die Unterschiede signifikant. Im geringsten Maße stehen Arbeitszeitkonten den geringfügig beschäftigten Frauen zur Verfügung, was ein erster Hinweis auf eine potenzielle Benachteiligung dieser Beschäftigtengruppe ist. ${ }^{7}$

\footnotetext{
${ }^{7}$ Aufgrund der relativ geringen Fallzahlen von geringfügig beschäftigten Männern mit einem Arbeitszeitkonto wird auf eine Darstellung verzichtet.
} 
Tabelle 4: Verbreitung von Arbeitszeitkonten nach Arbeitszeitumfang und Geschlecht

2002

\section{Vollzeit}

Männer

Frauen

Gesamt

Teilzeit

Männer

Frauen

Gesamt

Geringfügige Beschäftigung

Männer

Frauen

Gesamt

Quelle: SOEP, hochgerechnete Ergebnisse, eigene Berechnungen.

\begin{tabular}{llll}
43.7 & 49.3 & 49.7 & 54.8 \\
43.9 & 50.4 & 53.6 & 56.3 \\
43.8 & 49.6 & 51.1 & 55.3 \\
& & & \\
39.1 & 39.3 & 43.8 & 48.0 \\
34.4 & 45.1 & 46.9 & 52.6 \\
34.8 & 44.5 & & 52.2 \\
& & & \\
13.2 & 12.9 & 15.5 & 23.2 \\
12.6 & 12.6 & 14.5 & 21.6 \\
\hline
\end{tabular}

2009

2012

5.8

6.3

.3

8.0

2.6

2.2

3.2

1.6

\section{Ergebnisse der multivariaten Analyse}

Tabelle 5 und Tabelle 6 zeigen die Ergebnisse der logistischen Regressionsanalysen getrennt für Männer und Frauen. Aufgrund der geringen Fallzahlen wurde bei den Männern nicht für eine geringfügige Beschäftigung kontrolliert. ${ }^{8}$ Folgende Ergebnisse sind festzuhalten: Erstens haben geringfügig beschäftigte Frauen im Vergleich zu vollzeitbeschäftigten Frauen unterdurchschnittlich häufig Zugang zu einem Arbeitszeitkonto. Im Jahr 2012 reduziert sich das Risiko bzw. die Chance von Arbeitszeitkonten für geringfügig beschäftigte Frauen signifikant um rund 60 Prozent. Dieses Ergebnis kann zum einen auf die schlechtere Stellung von geringfügig Beschäftigten im Arbeitsmarkt zurückzuführen sein. Zum anderen spricht das Ergebnis dafür, dass die Arbeitszeiten von geringfügig Beschäftigten von betrieblicher Seite oft vorgegeben sind und eine Arbeitszeitverlängerung aufgrund der Verdienstgrenze nur bis zu einem gewissen Maße möglich ist. Teilzeitbeschäftigte Männer sind beim Zugang zu einem Arbeitszeitkonto gegenüber vollzeitbeschäftigten Männern nicht grundsätzlich benachteiligt, da sich keine signifikanten Unterschiede zeigen. Gesamtwirtschaftlich betrachtet sind unter den geringfügig Beschäftigten mehrheitlich Frauen zu finden. Dadurch stehen die Ergebnisse der Betriebsebene mit einem negativen Zusammenhang des Anteils von geringfügig Beschäftigten und Arbeitszeitkonten in Einklang mit den Ergebnissen der Individualebene.

Zweitens scheinen befristet beschäftigte Männer tendenziell unterdurchschnittlich häufig über Arbeitszeitkonten verfügen zu können als Beschäftigte mit einem dauerhaften Arbeitsvertrag. Allerdings ist der Effektkoeffizient für das Jahr 2009 nur schwach und für das Jahr 2012 nicht signifikant. Bei Frauen zeigt sich kein signifikanter Unterschied, wonach befristet gegenüber unbefristet beschäftigten Frauen nicht grundsätzlich benachteiligt sind. Auf der

\footnotetext{
${ }^{8}$ Jedoch haben sich auch bei Aufnahme der geringfügigen Beschäftigung keine Unterschiede in den Schätzergebnissen bei Männern gezeigt.
} 
Betriebsebene hat sich ein negativer Zusammenhang zwischen der Befristungsquote und der Wahrscheinlichkeit von Arbeitszeitkonten gezeigt. Dieser negative Zusammenhang kann auf der Individualebene insgesamt nicht bestätigt werden.

Drittens lässt sich bei beschäftigten Männern und Frauen in Leiharbeit kein unterschiedlicher Zugang zu einem Arbeitszeitkonto gegenüber Nicht-Leiharbeitnehmern erkennen. Danach sind Leiharbeiter nicht grundsätzlich vom Zugang zu einem Arbeitszeitkonto ausgeschlossen. Die Betriebsdaten weisen auf einen positiven Zusammenhang zwischen dem Leiharbeiteranteil und Arbeitszeitkonten hin, womit Betriebe mit einem hohen Flexibilitätsbedarf komplementär Arbeitszeitkonten und Leiharbeit nutzen und eine Polarisierung zwischen Stamm- und Randbelegschaften entsteht. Hierbei kann also angenommen werden, dass Leiharbeitnehmer ebenso wie Stammbeschäftigte über Arbeitszeitkonten verfügen, die Leiharbeit aber eine zusätzliche Pufferfunktion darstellt.

Viertens haben Beschäftigte mit einer benötigten abgeschlossenen Berufsausbildung zur Ausübung ihrer beruflichen Tätigkeit eine höhere Wahrscheinlichkeit des Zugangs zu einem Arbeitszeitkonto. Bezogen auf das Jahr 2012 erhöht sich das Risiko bzw. die Chance des Zugangs zu einem Arbeitszeitkonto für Männer mit abgeschlossener Berufsausbildung signifikant um rund 51 Prozent. Bei Frauen sind es entsprechend rund 53 Prozent. Bei diesen qualifizierten Beschäftigten kann ein höheres $\mathrm{Maß}$ an angesammeltem betriebsspezifischem $\mathrm{Hu}$ mankapital vermutet werden. Daher sind Arbeitgeber interessiert, das Humankapital dieser qualifizierten Beschäftigten möglichst intensiv zu nutzen, indem bei Auftragsspitzen die Arbeitszeit vorübergehend verlängert wird. Auch soll diese Beschäftigtengruppe bei negativen Nachfrageveränderungen im Betrieb gehalten und auf Entlassungen verzichtet werden. Eine weitere Erklärung ist, dass qualifizierte Beschäftigte eine höhere Marktmacht haben und sich gezielt Betriebe mit Arbeitszeitkonten als Arbeitgeber aussuchen können. Der positive Zusammenhang zwischen der abgeschlossenen Berufsausbildung und dem Zugang zu einem Arbeitszeitkonto steht in Einklang mit den Ergebnissen der Betriebsdaten. Mit den Betriebsdaten wurde gezeigt, dass mit einem zunehmenden Anteil qualifizierter Beschäftigter die Wahrscheinlichkeit von Arbeitszeitkonten in Betrieben steigt. Weiterhin weisen Frauen mit mittlerer beruflicher Autonomie überdurchschnittlich häufig Arbeitszeitkonten auf, dagegen haben Männer mit einer eher hohen Autonomie tendenziell unterdurchschnittlich häufig Zugang zu einem Arbeitszeitkonto. Der negative Zusammenhang lässt sich damit begründen, dass Männer mit eher hoher beruflicher Autonomie häufiger andere Arbeitszeitarrangements aufweisen. Sie leisten eher unbezahlte Überstunden und/oder haben Vertrauensarbeitszeit, die Arbeitszeitkonten entgegen stehen. 
Tabelle 5: Determinanten des Zugangs zu einem Arbeitszeitkonto, Männer

\begin{tabular}{|c|c|c|c|c|c|c|c|c|c|}
\hline \multirow{4}{*}{$\begin{array}{l}\text { Vertragl. Arbeitszeit (Ref:: Vollze } \\
\text { Teilzeit }\end{array}$} & \multirow{2}{*}{\multicolumn{2}{|c|}{$\begin{array}{l}2006 \\
\text { Odds } \\
\text { Ratio }\end{array}$}} & \multirow{3}{*}{$\begin{array}{r}\text { Std.- } \\
\text { fehler }\end{array}$} & \multicolumn{2}{|l|}{2009} & \multicolumn{4}{|c|}{2012} \\
\hline & & & & \multicolumn{2}{|l|}{$\begin{array}{l}\text { Odds } \\
\text { Ratio }\end{array}$} & \multirow[t]{2}{*}{$\begin{array}{l}\text { Std.- } \\
\text { fehler }\end{array}$} & \multicolumn{2}{|l|}{$\begin{array}{l}\text { Odds } \\
\text { Ratio }\end{array}$} & \multirow[t]{2}{*}{$\begin{array}{r}\text { Std.- } \\
\text { fehler }\end{array}$} \\
\hline & & & & & & & & & \\
\hline & 1.163 & & 0.246 & 1.068 & & 0.200 & 0.990 & & 0.200 \\
\hline \multicolumn{10}{|l|}{ Geringfügige Beschäftigung } \\
\hline Befristet & 0.657 & $* * *$ & 0.095 & 0.779 & * & 0.104 & 0.990 & & 0.144 \\
\hline Leiharbeit & 1.375 & & 0.287 & 0.958 & & 0.198 & 1.417 & & 0.336 \\
\hline \multicolumn{10}{|c|}{$\begin{array}{l}\text { Ausbildung (Ref:: Keine bes. Ausbil- } \\
\text { dung) }\end{array}$} \\
\hline Abgeschl. Berufsausbildung & 1.759 & $* * *$ & 0.172 & 1.417 & $* * *$ & 0.138 & 1.510 & $* * *$ & 0.168 \\
\hline Abgeschl. Hochschulstudium & 1.268 & * & 0.174 & 1.083 & & 0.151 & 1.139 & & 0.173 \\
\hline \multicolumn{10}{|l|}{$\begin{array}{l}\text { Autonomie (Ref:: Eher niedrige } \\
\text { Autonomie) }\end{array}$} \\
\hline Mittlere Autonomie & 0.949 & & 0.114 & 1.195 & & 0.131 & 1.057 & & 0.132 \\
\hline Eher hohe Autonomie & 0.578 & $* * *$ & 0.077 & 0.720 & ** & 0.093 & 0.819 & & 0.117 \\
\hline \multicolumn{10}{|l|}{ Berufliche Stellung (Ref:: Arbeiter) } \\
\hline Angestellter & 0.933 & & 0.111 & 0.692 & $* * *$ & 0.075 & 0.625 & $* * *$ & 0.075 \\
\hline Beamter & 1.051 & & 0.194 & 0.859 & & 0.154 & 0.766 & & 0.153 \\
\hline \multicolumn{10}{|l|}{$\begin{array}{l}\text { Einarbeitung (Ref:: Kurze Einwei- } \\
\text { sung) }\end{array}$} \\
\hline Längere Einarbeitung & 1.167 & * & 0.109 & 1.419 & $* * *$ & 0.137 & 1.270 & $* *$ & 0.134 \\
\hline Lehrgänge/Kurse & 1.337 & $* * *$ & 0.137 & 1.557 & $* * *$ & 0.162 & 1.454 & $* * *$ & 0.163 \\
\hline \multicolumn{10}{|c|}{$\begin{array}{l}\text { Betriebsgröße (Ref.: < } 20 \text { Beschäftig- } \\
\text { te) }\end{array}$} \\
\hline 20 bis 199 Beschäftigte & 1.287 & $* *$ & 0.132 & 1.271 & ** & 0.126 & 1.436 & $* * *$ & 0.165 \\
\hline 200 bis 1999 Beschäftigte & 1.631 & $* * *$ & 0.175 & 1.666 & $* * *$ & 0.177 & 1.861 & $* * *$ & 0.222 \\
\hline Über 2000 Beschäftigte & 1.923 & $* * *$ & 0.206 & 2.022 & $* * *$ & 0.211 & 1.903 & $* * *$ & 0.225 \\
\hline \multicolumn{10}{|l|}{$\begin{array}{l}\text { Wirtschaftsbereich (Ref.: Prod. } \\
\text { Gewerbe inkl. Bau) }\end{array}$} \\
\hline Handel/Verkehr/Gastgewerbe & 0.744 & $* * *$ & 0.075 & 0.656 & $* * *$ & 0.064 & 0.761 & $* * *$ & 0.081 \\
\hline Sonstige Dienstleistungen & 0.769 & $* * *$ & 0.077 & 0.842 & * & 0.082 & 0.943 & & 0.101 \\
\hline $\begin{array}{l}\text { Öff. Dienstleis- } \\
\text { ter/Erziehung/Gesundheit }\end{array}$ & 1.052 & & 0.121 & 1.131 & & 0.127 & 1.119 & & 0.137 \\
\hline Ostdeutschland & 1.382 & $* * *$ & 0.115 & 1.345 & $* * *$ & 0.105 & 1.203 & $* *$ & 0.104 \\
\hline Konstante & 0.469 & $* * *$ & 0.058 & 0.499 & $* * *$ & 0.062 & 0.590 & $* * *$ & 0.083 \\
\hline Fallzahl & 3971 & & & 4123 & & & 3348 & & \\
\hline $\begin{array}{l}\text { Pseudo- } \mathrm{R}^{2} \text { (McKelvey and } \\
\text { Zavoina's) }\end{array}$ & 0.03 & & & 0.04 & & & 0.03 & & \\
\hline Pseudo-R ${ }^{2}$ (McFadden) & 0.04 & & & 0.04 & & & 0.03 & & \\
\hline
\end{tabular}

Quelle: SOEP, hochgerechnete Ergebnisse, eigene Berechnungen. 
Tabelle 6: Determinanten des Zugangs zu einem Arbeitszeitkonto, Frauen

\begin{tabular}{|c|c|c|c|c|c|c|c|c|c|}
\hline \multirow{3}{*}{ Vertragl. Arbeitszeit (Ref.: Vollzei } & \multicolumn{3}{|l|}{2006} & \multicolumn{3}{|l|}{2009} & \multicolumn{3}{|l|}{2012} \\
\hline & \multicolumn{2}{|l|}{$\begin{array}{l}\text { Odds } \\
\text { Ratio }\end{array}$} & \multirow[t]{2}{*}{$\begin{array}{l}\text { Std.- } \\
\text { fehler }\end{array}$} & \multicolumn{2}{|l|}{$\begin{array}{l}\text { Odds } \\
\text { Ratio }\end{array}$} & \multirow[t]{2}{*}{$\begin{array}{l}\text { Std.- } \\
\text { fehler }\end{array}$} & \multicolumn{2}{|l|}{$\begin{array}{l}\text { Odds } \\
\text { Ratio }\end{array}$} & \multirow[t]{2}{*}{$\begin{array}{l}\text { Std.- } \\
\text { fehler }\end{array}$} \\
\hline & & & & & & & & & \\
\hline Teilzeit & 0.960 & & 0.073 & 0.857 & $* *$ & 0.062 & 0.932 & & 0.072 \\
\hline Geringfügige Beschäftigung & 0.362 & $* * *$ & 0.079 & 0.354 & $* * *$ & 0.065 & 0.402 & $* * *$ & 0.074 \\
\hline Befristet & 1.003 & & 0.139 & 1.073 & & 0.130 & 0.848 & & 0.105 \\
\hline Leiharbeit & 1.178 & & 0.328 & 0.969 & & 0.248 & 0.922 & & 0.244 \\
\hline \multicolumn{10}{|c|}{$\begin{array}{l}\text { Ausbildung (Ref:: Keine bes. Ausbil- } \\
\text { dung) }\end{array}$} \\
\hline Abgeschl. Berufsausbildung & 1.557 & $* * *$ & 0.178 & 1.571 & $* * *$ & 0.179 & 1.530 & $* * *$ & 0.175 \\
\hline Abgeschl. Hochschulstudium & 0.953 & & 0.152 & 1.060 & & 0.171 & 1.048 & & 0.170 \\
\hline \multicolumn{10}{|l|}{$\begin{array}{l}\text { Autonomie (Ref.: Eher niedrige } \\
\text { Autonomie) }\end{array}$} \\
\hline Mittlere Autonomie & 1.203 & * & 0.122 & 1.175 & * & 0.114 & 1.396 & $* * *$ & 0.147 \\
\hline Eher hohe Autonomie & 0.965 & & 0.141 & 1.005 & & 0.146 & 1.168 & & 0.179 \\
\hline \multicolumn{10}{|l|}{ Berufliche Stellung (Ref.: Arbeiter) } \\
\hline Angestellter & 1.291 & $* *$ & 0.166 & 1.079 & & 0.131 & 1.201 & & 0.158 \\
\hline Beamter & 0.766 & & 0.164 & 0.590 & $* * *$ & 0.117 & 0.698 & * & 0.148 \\
\hline \multicolumn{10}{|l|}{$\begin{array}{l}\text { Einarbeitung (Ref:: Kurze Einwei- } \\
\text { sung) }\end{array}$} \\
\hline Längere Einarbeitung & 1.455 & $* * *$ & 0.130 & 1.304 & $* * *$ & 0.118 & 1.342 & $* * *$ & 0.127 \\
\hline Lehrgänge/Kurse & 1.319 & $* * *$ & 0.138 & 1.464 & $* * *$ & 0.150 & 1.351 & $* * *$ & 0.144 \\
\hline \multicolumn{10}{|c|}{$\begin{array}{l}\text { Betriebsgröße (Ref.: < } 20 \text { Beschäftig- } \\
\text { te) }\end{array}$} \\
\hline 20 bis 199 Beschäftigte & 1.514 & $* * *$ & 0.147 & 1.855 & $* * *$ & 0.175 & 1.542 & $* * *$ & 0.159 \\
\hline 200 bis 1999 Beschäftigte & 1.942 & $* * *$ & 0.205 & 2.648 & $* * *$ & 0.270 & 2.047 & $* * *$ & 0.223 \\
\hline Über 2000 Beschäftigte & 1.629 & $* * *$ & 0.172 & 2.425 & $* * *$ & 0.250 & 2.158 & $* * *$ & 0.232 \\
\hline \multicolumn{10}{|l|}{$\begin{array}{l}\text { Wirtschaftsbereich (Ref.: Prod. } \\
\text { Gewerbe inkl. Bau) }\end{array}$} \\
\hline Handel/Verkehr/Gastgewerbe & 0.745 & $* *$ & 0.091 & 0.892 & & 0.106 & 0.796 & * & 0.100 \\
\hline Sonstige Dienstleistungen & 0.830 & & 0.098 & 0.979 & & 0.111 & 0.962 & & 0.116 \\
\hline $\begin{array}{l}\text { Öff. Dienstleis- } \\
\text { ter/Erziehung/Gesundheit }\end{array}$ & 1.133 & & 0.122 & 1.365 & $* * *$ & 0.143 & 1.329 & $* * *$ & 0.145 \\
\hline Ostdeutschland & 1.162 & * & 0.099 & 1.197 & $* *$ & 0.097 & 1.079 & & 0.092 \\
\hline Konstante & 0.306 & $* * *$ & 0.045 & 0.248 & $* * *$ & 0.036 & 0.304 & $* * *$ & 0.049 \\
\hline Fallzahl & 3511 & & & 3873 & & & 3382 & & \\
\hline $\begin{array}{l}\text { Pseudo- } \mathrm{R}^{2} \text { (McKelvey and } \\
\text { Zavoina's) }\end{array}$ & 0.04 & & & 0.05 & & & 0.05 & & \\
\hline Pseudo- $\mathrm{R}^{2}$ (McFadden) & 0.06 & & & 0.07 & & & 0.07 & & \\
\hline
\end{tabular}

Quelle: SOEP, hochgerechnete Ergebnisse, eigene Berechnungen.

In Hinblick auf die Kontrollvariablen zeigt sich, dass Männer in einem Angestelltenverhältnis unterdurchschnittlich häufig Zugang zu einem Arbeitszeitkonto im Vergleich zu Arbeitern haben. Bei Frauen verfügen tendenziell Beamtinnen unterdurchschnittlich häufig über Ar- 
beitszeitkonten. Bei beiden Geschlechtern zeigt sich, dass Beschäftigte mit einer größeren Quantität und Qualität der erforderlichen Einarbeitung überdurchschnittlich häufig ein Arbeitszeitkonto haben im Vergleich zu Beschäftigten mit nur einer kurzen Einweisung in die Tätigkeit. Im Jahr 2012 erhöht sich das Risiko bzw. die Chance des Zugangs zu einem Arbeitszeitkonto für Männer mit einer längeren Einarbeitung um rund 27 Prozent und für Frauen um rund 34 Prozent. Bei einer längeren und anspruchsvolleren Einarbeitungsphase kann davon ausgegangen werden, dass Beschäftigte in höherem Maße über betriebsspezifisches $\mathrm{Hu}$ mankapital verfügen. Daher sind Arbeitgeber an Arbeitszeitkonten interessiert und stellen diese bereit. Männer und Frauen, die in größeren Betrieben beschäftigt sind, haben überdurchschnittlich häufig Arbeitszeitkonten im Vergleich zu Beschäftigten in kleineren Betrieben mit weniger als 20 Mitarbeitern. Männer, die in den Branchen Handel, Verkehr und Gastgewerbe und in den Sonstigen Dienstleistungen tätig sind haben weniger häufig Zugang zu einem Arbeitszeitkonto als Beschäftigte im Produzierenden Gewerbe inklusive dem Baugewerbe. Frauen im Handel, Verkehr und Gastgewerbe haben ebenfalls unterdurchschnittlich häufig Zugang zu einem Arbeitszeitkonto, während Frauen im Bereich Öffentliche Dienstleister, Erziehung und Gesundheit tendenziell häufiger über Arbeitszeitkonten verfügen. Männer in Ostdeutschland haben überdurchschnittlich häufig ein Arbeitszeitkonto. Bei Frauen ist der Effektkoeffizient in 2006 nur schwach und in 2009 nicht signifikant. Insgesamt betrachtet stehen die Ergebnisse zu Betriebsgröße, Branchen und Region auf der Individualebene in Einklang mit den aggregierten Ergebnissen der Betriebsebene.

\section{Zusammenfassung}

Arbeitszeitkonten sind in Deutschland inzwischen weit verbreitet. Sie sind ein internes Flexibilitätsinstrument, um die Arbeitszeiten von Beschäftigten zu variieren und den Arbeitseinsatz an das Arbeitsaufkommen anzupassen. Dabei werden die Abweichungen der tatsächlichen von der vertraglich vereinbarten Arbeitszeit auf den Arbeitszeitkonten verbucht und in einem bestimmten Bezugszeitraum wieder ausgeglichen. Arbeitszeitkonten bieten eine Substitutionsmöglichkeit zu externer Flexibilität, um Beschäftigung weitgehend zu sichern. Die Ergebnisse der Modellschätzungen auf der Betriebsebene zeigen, dass die industriellen Beziehungen und die betriebliche Beschäftigungsstruktur zentrale Bestimmungsgründe für Arbeitszeitkonten sind. Diese Bestimmungsgründe sind auch im Zeitverlauf konstant, d. h. die Bestimmungsfaktoren ändern sich im Zeitverlauf nicht, obwohl sich die verschiedenen Zeitpunkte durch unterschiedliche konjunkturelle Lagen auszeichnen. Dies weist darauf hin, dass Arbeitszeitkonten nicht kurzfristig eingeführt werden, um in einer konjunkturellen Auf- oder Abschwungphase die Arbeitszeiten der Beschäftigten kurzfristig nach oben oder unten anzupassen. Vielmehr unterliegen Arbeitszeitkonten einem längeren Entscheidungsprozess zwischen Arbeitgebern bzw. ihren Interessenvertretern und den Gewerkschaften sowie Betriebs/Personalräten. Auf beiden Seiten sind organisatorische und bürokratische Bemühungen notwendig, damit Arbeitszeitkonten im Betrieb implementiert werden.

Die Ergebnisse der Modellschätzungen auf der Beschäftigtenebene fallen bei Männern und Frauen zum Teil unterschiedlich aus. Auch gibt es eine zeitliche Variation der Bestimmungsfaktoren. So haben geringfügig beschäftigte Frauen im Vergleich zu vollzeitbeschäftigten 
Frauen unterdurchschnittlich häufig Zugang $\mathrm{zu}$ einem Arbeitszeitkonto. Dieser ungleiche Zugang kann zum einen auf die schlechtere Stellung am Arbeitsmarkt zurückzuführen sein, zum anderen lohnen sich Arbeitszeitkonten aus betrieblicher Sicht bei geringfügig Beschäftigten nicht. Tendenziell scheinen befristet beschäftigte Männer gegenüber unbefristet Beschäftigten unterdurchschnittlich häufig Zugang zu einem Arbeitszeitkonto zu haben. Dies kann ebenfalls darauf zurückzuführen sein, dass befristet beschäftigte Männer, wie geringfügig beschäftigte Frauen, eine schlechtere Stellung im Arbeitsmarkt haben und deshalb beim Zugang zu einem Arbeitszeitkonto benachteiligt sind. Bei beiden Geschlechtern zeigt sich, dass eine benötigte abgeschlossene Berufsausbildung zur Ausübung der beruflichen Tätigkeit positiv mit dem Zugang zu einem Arbeitszeitkonto korreliert. Die Humankapitalausstattung eines Beschäftigten trägt also wesentlich zur Erklärung des Zugangs zu Arbeitszeitkonten von Beschäftigten bei. Die hohe Bedeutung des Humankapitals zeigt sich auch beim positiven $\mathrm{Zu}-$ sammenhang zwischen der Quantität und Qualität der erforderlichen Einarbeitung und dem Zugang zu einem Arbeitszeitkonto. Die Bestimmungsgründe des Zugangs zu einem Arbeitszeitkonto von Beschäftigten weisen ebenfalls eine hohe zeitliche Konstanz auf. Diese hohe zeitliche Konstanz spricht auch für robuste Ergebnisse.

Insgesamt zeigen die Ergebnisse auf der Beschäftigtenseite, dass gut integrierte Beschäftigte im Arbeitsmarkt auch überdurchschnittlich häufig ein Arbeitszeitkonto haben, während dies für benachteiligte Beschäftigtengruppen, wie geringfügig beschäftigte Frauen und befristet beschäftigte Männer, nicht gilt. Arbeitszeitkonten als internes Flexibilitätsinstrument tragen somit zu einer weiteren Spaltung am Arbeitsmarkt zwischen Insidern mit stabilen und gut geschützten Beschäftigungsverhältnissen und Outsidern mit unsicheren und weniger gut geschützten Beschäftigungsverhältnissen bei. Allerdings sind die potenziellen Risiken und Nachteile von Arbeitszeitkonten für Beschäftigte nicht zu vernachlässigen. So können langfristig geleistete Mehrarbeitsstunden ohne (zeitnahen) Freizeitausgleich faktisch zu bezahlten und unbezahlten Überstunden werden. Überstunden können die Work-Life-Balance der Beschäftigten negativ beeinflussen und $\mathrm{zu}$ gesundheitlichen Problemen führen (Goudswaard et al. 2009).

Vor diesem Hintergrund ist es arbeits- und gesellschaftspolitisch notwendig weitere Forschungsfragen empirisch zu adressieren, die die potenziellen Risiken und Nachteile von Arbeitszeitkonten aufgreifen. So ist bislang unklar in welchem Umfang Guthaben auf Arbeitszeitkonten angesammelt werden und ob diese tatsächlich rechtzeitig ausgeglichen werden können. In diesem Kontext ist weiterhin zu analysieren, für welche Zwecke die angesammelten Guthaben auf Arbeitszeitkonten tatsächlich genutzt werden und inwieweit es Zielkonflikte zwischen betrieblichen und individuellen Bedürfnissen gibt. Eine weitere offene Forschungsfrage ist, ob und wie Arbeitszeitkonten auf die Arbeitszeitautonomie und Zufriedenheit der Beschäftigten wirken. Zur Beantwortung dieser Fragen ist es notwendig bislang bestehende Datenlücken zu schließen, um Arbeitszeitkonten in der betrieblichen Praxis weiter beurteilen zu können.

\section{Literaturverzeichnis}

Anxo, Dominique und Jean-Yves Boulin (2006). The Organization of Time Over the Life Course. European Trends. European Societies, 8(2), 319-341. 
Arlinghaus, Anna und Friedhelm Nachreiner (2012). Arbeit zu unüblichen Zeiten - Arbeit mit unüblichem Risiko. Zeitschrift für Arbeitswissenschaft, 66(4), 291-305.

Bauer, Frank, Hermann Groß, Eva Munz und Suna Sayin (2002). Arbeits- und Betriebszeiten 2001: Neue Formen des betrieblichen Arbeits- und Betriebszeitmanagements. Ergebnisse einer repräsentativen Betriebsbefragung. Köln.

Bauer, Frank, Hermann Groß, Klaudia Lehmann und Eva Munz (2004). Arbeitszeit 2003: Arbeitszeitgestaltung, Arbeitsorganisation und Tätigkeitsprofile. Köln.

Bellmann, Lutz (2002). Das IAB-Betriebspanel: Konzeption und Anwendungsbereiche. Allgemeines Statistisches Archiv, 86, 177-188.

Bellmann, Lutz und Tilo Gewiese (2003). Betriebliche Arbeitszeitstrukturen in der Bundesrepublik Deutschland: Empirische Ergebnisse aus dem IAB-Betriebspanel 1996-1999 unter besonderer Berücksichtigung der Arbeitszeitflexibilisierung. Dortmund/Berlin/Dresden: Wirtschafsverlag NW.

Bellmann, Lutz und Tilo Gewiese (2004). Die Dynamik der Nutzung von Arbeitszeitkonten: Theoretische Ansätze und Längsschnittanalysen mit dem IAB-Betriebspanel. In: Bellmann, Lutz und Claus Schnabel (Hg.). Betriebliche Arbeitszeitpolitik im Wandel. Beiträge zur Arbeitsmarkt- und Berufsforschung 288 (139-184). Nürnberg.

Bellmann, Lutz und Hans-Dieter Gerner (2011). Reversed Roles? Wage and Employment Effects of the Current Crisis. In: Immervoll, Herwig, Andreas Peichl und Konstantinos Tatsiramos (Hg.). Who Loses in the Downturn? Economic Crisis, Employment and Income Distribution (Research in Labor Economics, 32) (181-206). Bingley: Emerald.

Bellmann, Lutz, Hans-Dieter Gerner und Richard Upward (2012). The Response of German Establishments to the 2008-2009 Economic Crisis. OECD Social, Employment and Migration Working Papers 137. Paris: OECD.

Berg, Peter (2008). Working Time Flexibility in the German Employment Relations System: Implications for Germany and Lessons for the United States. Industrielle Beziehungen, 15(2), 133-150.

Best, Henning und Christof Wolf (2010). Logistische Regression. In: Wolf, Christof und Henning Best (Hg.). Handbuch der sozialwissenschaftlichen Datenanalyse (827-854). Wiesbaden: VS Verlag für Sozialwissenschaften.

Bispinck, Reinhard (1998). Zwischen betrieblicher Flexibilität und Zeitsouveränität: Tarifliche Regelungen zu Arbeitszeitkonten. In: Klenner, Christina und Hartmut Seifert (Hg.). Zeitkonten - Arbeit à la carte? Neue Modelle der Arbeitszeitgestaltung (72-87). Hamburg: VSA Verlag.

Bispinck, Reinhard (2014). Tarifliche Arbeitszeitregelungen zwischen betrieblichen Flexi-Ansprüchen und individuellen Arbeitszeitoptionen: Eine Analyse ausgewählter Tarifbereiche. Elemente qualitativer Tarifpolitik Nr. 79. Düsseldorf.

Boeri, Tito und Herbert Brücker (2011). Short-time Work Benefits Revisited: Some Lessons from the Great Recession. Economic Policy, 26(68), 697-765.

Bosch, Gerhard (1996a). Jahresarbeitszeiten: Flexibilität auch für Stammbelegschaften. Journal für Sozialforschung, 36(1), 3-22.

Bosch, Gerhard (1996b): Flexibilisierung der Arbeitszeit und Umverteilung von Arbeit. WSIMitteilungen, 49(7), 423-432. 
Bosch, Aida, Peter Ellguth und Markus Promberger (1992). Gleitzeit: Wieviel Zeitautonomie ist gefragt? WSI-Mitteilungen, 45(1), 51-59.

Bosch, Gerhard, Sebastian Schief und Marc Schietinger (2005). Trends in der Arbeitszeitpolitik: Zur Diskussion um Dauer und Flexibilisierung der Arbeitszeit sowie der Insolvenzsicherung von Arbeitszeitguthaben. Bonn: Wirtschafts- und sozialpolitisches Forschungs- und Beratungszentrum der Friedrich-Ebert-Stiftung, Abteilung Arbeit und Sozialpolitik.

Bundesmann-Jansen, Jörg, Hermann Groß und Eva Munz (2000). Arbeitszeit '99: Ergebnisse einer repräsentativen Beschäftigtenbefragung zu traditionellen und neuen Arbeitszeitformen in der Bundesrepublik Deutschland. Köln.

Burda, Michael C. und Jennifer Hunt (2011). What Explains the German Labor Market Miracle in the Great Recession? Brookings Papers on Economic Activity, 42(1), 273-335.

Carstensen, Vivian (1999). Alternative Flexibilisierungsstrategien der Beschäftigung. Diskussionspapier Nr. 229. Hannover: Fachbereich Wirtschaftswissenschaften, Universität Hannover.

Carstensen, Vivian (2000). Arbeitsplatzsicherheit durch Arbeitszeitkonten? In: Backes-Gellner, Uschi, Matthias Kräkel, Bernd Schauenberg und Gunter Steiner (Hg.). Flexibilisierungstendenzen in der betrieblichen Personalpolitik. Anreize, Arbeitszeiten und Qualifikation (307-332). München/Mering: Rainer Hampp Verlag.

Dietz, Martin, Michael Stops und Ulrich Walwei (2011). Safeguarding Jobs in Times of Crisis: Lessons From the German Experience. IILS Discussion Paper 207. Geneva: International Institute for Labour Studies.

Ellguth, Peter, Hans-Dieter Gerner und Ines Zapf (2013). Flexibilität für Betriebe und Beschäftigte: Vielfalt und Dynamik bei den Arbeitszeitkonten. IAB-Kurzbericht 3. Nürnberg.

Ellguth, Peter und Markus Promberger (2004). Arbeitszeitsituation und Betriebsrat - eine MatchedPair-Analyse mit Daten des IAB-Betriebspanels. In: Bellmann, Lutz und Claus Schnabel (Hg.). Betriebliche Arbeitszeitpolitik im Wandel. Beiträge zur Arbeitsmarkt- und Berufsforschung 288 (111131). Nürnberg.

Esser, Ingo (2007). Arbeitszeitkonten und Altersvorsorge: Chancen für Arbeitgeber und Arbeitnehmer. Saarbrücken: VDM Verlag Dr. Müller.

Fischer, Gabriele, Florian Janik, Dana Müller und Alexandra Schmucker (2009). The IAB Establishment Panel - Things Users Should Know. Schmollers Jahrbuch, 129, 133-148.

Göbel, Jan, Peter Krause, Rainer Pischner, Ingo Sieber und Gerd G. Wagner (2008). Daten- und Datenbankstruktur der Längsschnittstudie Sozio-oekonomisches Panel (SOEP). SOEPpapers on Multidisciplinary Panel Data Research 89. Berlin: DIW.

Goudswaard, Anneke, Peter Oeij, Tony Brugman und Tanja de Jong (2009). Good Practice Guide to Internal Flexibility Policies in Companies. Dublin: European Foundation for the Improvement of Living and Working Conditions.

Greene, William H. (2008). Econometric Analysis. Upper Saddle River: Pearson Prentice Hall.

Greubel, Jana, Anna Arlinghaus und Friedhelm Nachreiner (2014). Arbeitszeitflexibilität auf Kosten der Sicherheit? Zum Zusammenhang zwischen der Variabilität der Arbeitszeit und der Häufigkeit von Arbeitsunfällen. Zeitschrift für Arbeitswissenschaft, 68(2), 89-95. 
Groß, Hermann (2009). Vergleichende Analyse der Arbeits- und Betriebszeitentwicklung im Zeitraum von 1987 bis 2007. Dortmund: Sozialforschungsstelle, Technische Universität Dortmund.

Groß, Hermann und Michael Schwarz (2006). Betriebs- und Arbeitszeiten 2005: Ergebnisse einer repräsentativen Betriebsbefragung. Dortmund: Sozialforschungsstelle, Technische Universität Dortmund.

Groß, Hermann und Michael Schwarz (2008). Arbeitszeitarrangements, Altersstrukturen und Corporate Social Responsibility in deutschen Betrieben: Ergebnisse einer in 2007 durchgeführten repräsentativen Betriebsbefragung. Dortmund: Sozialforschungsstelle, Technische Universität Dortmund.

Groß, Hermann und Michael Schwarz (2010). Arbeitszeit, Altersstrukturen und Corporate Social Responsibility: Eine repräsentative Betriebsbefragung. Wiesbaden: VS Verlag für Sozialwissenschaften.

Herzog-Stein, Alexander und Ines Zapf (2014). Navigating the Great Recession: The Impact of Working-Time Accounts in Germany. Industrial \& Labor Relations Review, 67(3), 891-925.

Hildebrandt, Eckart (2006). Balance Between Work and Life - New Corporate Impositions Through Flexible Working Time or Opportunity for Time Sovereignty? European Societies, 8(2), 251-271.

Hildebrandt, Eckart (2007). Langzeitkonten, Lebensplanung und Zeithandeln. In: Hildebrandt, Eckart (Hg.): Lebenslaufpolitik im Betrieb: Optionen zur Gestaltung der Lebensarbeitszeit durch Langzeitkonten (173-202). Berlin: Edition Sigma.

Hildebrandt, Eckart, Philip Wotschack und Almut Kirschbaum (2009). Zeit auf der hohen Kante: Langzeitkonten in der betrieblichen Praxis und Lebensgestaltung von Beschäftigten. Berlin: Edition Sigma.

Hill, E. Jeffrey, Alan J. Hawkins, Maria Ferris und Michelle Weitzman (2001). Finding an Extra Day a Week: The Positive Influence of Perceived Job Flexibility on Work and Family Life Balance. Family Relations, 50(1), 49-58.

Hinrichs, Karl (1992). Zur Zukunft der Arbeitszeitflexibilisierung. Arbeitnehmerpräferenzen, betriebliche Interessen und Beschäftigungswirkungen. Soziale Welt, 43(3), 313-330.

Hinrichs, Karl und Helmut Wiesenthal (1982). Arbeitswerte und Arbeitszeit: Zur Pluralisierung von Wertmustern und Zeitverwendungswünschen in der modernen Industriegesellschaft. In: Offe, Claus, Karl Hinrichs und Helmut Wiesenthal (Hg.). Arbeitszeitpolitik. Formen und Folgen einer Neuverteilung der Arbeitszeit (116-136). Frankfurt: Campus Verlag.

Hübler, Olaf und Wolfgang Meyer (1997). Überstunden im Verarbeitenden Gewerbe Niedersachsens. In: Kühl, Jürgen, Manfred Lahner und Joachim Wagner (Hg.). Die Nachfrageseite des Arbeitsmarktes: Ergebnisse aus den Analysen mit deutschen Firmenpaneldaten. Beiträge zur Arbeitsmarkt- und Berufsforschung 204 (227-254). Nürnberg.

Janßen, Daniela und Friedhelm Nachreiner (2004). Flexible Arbeitszeiten. Schriftenreihe Fb 1025. Dortmund/Berlin/Dresden: Bundesanstalt für Arbeitsschutz und Arbeitsmedizin.

Knutsson, Anders (2003): Health disorders of shift workers. Journal of Occupational Medicine and Toxicology, 53, 103-108.

Kraetsch, Clemens und Rainer Trinczek (1998). Arbeitszeitflexibilisierung in Klein- und Mittelbetrieben des Produzierenden Gewerbes: Stand, Entwicklung und Probleme. Arbeit, 7(4), 338-361. 
Ludewig, Oliver (2001). Betriebliche Bestimmungsgründe von Arbeitszeitkonten. Mitteilungen aus der Arbeitsmarkt- und Berufsforschung, 34(3), 302-313.

Möller, Joachim (2010). The German Labor Market Response in the World Recession: De-Mystifying a Miracle. Zeitschrift für Arbeitsmarktforschung, 42(4), 325-336.

Pannenberg, Markus und Gert G. Wagner (2001). Overtime Work, Overtime Compensation and the Distribution of Economic Well-Being: Evidence for West Germany and Great Britain. IZA Discussion Paper 318. Bonn: Institute for the Study of Labour.

Paridon, Hiltraut, Sabine Ernst, Volker Harth, Peter Nickel, Annette Nold und Dirk Pallapies (2012). Schichtarbeit: Rechtslage, gesundheitliche Risiken und Präventionsmöglichkeiten. Report 1/2012. Berlin: DGUV.

Promberger, Markus, Sabine Böhm, Thilo Heyder, Susanne Pamer und Katharina Strauß (2002). Hochflexible Arbeitszeiten in der Industrie: Chancen, Risiken und Grenzen für Beschäftigte. Berlin: Edition Sigma.

Schief, Sebastian (2003). Arbeitszeiten in Deutschland - Eine Frage der Messung? Mitteilungen aus der Arbeitsmarkt- und Berufsforschung, 36(2), 187-198.

Seifert, Hartmut (1987). Variable Arbeitszeitgestaltung - Arbeitszeit nach Maß für die Betriebe oder Zeitautonomie für die Arbeitnehmer? WSI-Mitteilungen, 40(12), 727-735.

Seifert, Hartmut (1991). Mehr Zeitsouveränität durch variable Arbeitszeitgestaltung? In: Semlinger, Klaus (Hg.). Flexibilisierung des Arbeitsmarktes. Interessen, Wirkungen, Perspektiven (249-269). Frankfurt am Main/New York: Campus Verlag.

Seifert, Hartmut (1996). Arbeitszeitkonten - Modelle für mehr Zeitsouveränität oder absatzorientiertes Zeitmanagement? WSI-Mitteilungen, 49(7), 442-449.

Seifert, Hartmut (1998). Modellwechsel durch Arbeitszeitkonten. In: Klenner, Christina und Hartmut Seifert (Hg.). Zeitkonten - Arbeit à la carte? Neue Modelle der Arbeitszeitgestaltung (9-26). Hamburg: VSA Verlag.

Seifert, Hartmut (2001). Zeitkonten: Von der Normalarbeitszeit zu kontrollierter Flexibilität. WSIMitteilungen, 54(2), 334-340.

Seifert, Hartmut (2005). Vom Gleitzeit- zum Langzeitkonto. WSI-Mitteilungen, 58(6), 308-313.

Seifert, Hartmut, Angelika Kümmerling und Arnold Riedmann (2013). Langzeitkonten - überschätzte Erwartungen einer biografieorientierten Zeitpolitik? WSI-Mitteilungen, 66(2), 133-143.

Seifert, Hartmut (2014). Arbeitszeitkonten - Ein Ansatz für lebenslauforientierte Zeitgestaltung? Zeitschrift für Arbeitswissenschaft, 68 (2), 67-71.

Teriet, Bernhard (1976). „Zeitsouveränität“ durch flexible Arbeitszeit. Aus Politik und Zeitgeschichte, 31, 3-16.

Teriet, Bernhard (1977). Die Wiedergewinnung der Zeitsouveränität. Technologie und Politik - Das Magazin zur Wachstumskrise, 8, 75-111.

Wagner, Gert G., Joachim R. Frick und Jürgen Schupp (2007). The German Socio-Economic Panel Study (SOEP) - Scope, Evolution and Enhancements. Schmollers Jahrbuch, 127(1), 139-169.

Wellisch, Dietmar (2014). Mehr Mut zu Wertguthaben. Personalwirtschaft, 7, 18-20. 
Wolf, Elke und Miriam Beblo (2004). Arbeitszeitflexibilisierung und Effizienz in deutschen Unternehmen. In: Bellmann, Lutz und Claus Schnabel (Hg.). Betriebliche Arbeitszeitpolitik im Wandel. Beiträge zur Arbeitsmarkt- und Berufsforschung 288 (11-32). Nürnberg.

Wooldridge, Jeffrey M. (2013). Introductory Econometrics: A Modern Approach. Florence: SouthWestern Cengage Learning.

Wotschack, Philip (2010). Lebenslauforientierte Arbeitszeitgestaltung in Zeiten der Krise: Ansätze, Nutzungsbarrieren und Handlungsfelder. In: Groß, Hermann und Hartmut Seifert (Hg.). Zeitkonflikte: Renaissance der Arbeitszeitpolitik. Forschung aus der Hans-Böckler-Stiftung, Bd 115 (1952012). Berlin: Edition Sigma.

Zapf, Ines (2016). Traditionelle und moderne Formen der Arbeitszeitflexibilität: Arbeitsangebots- und -nachfrageseitige Faktoren von Überstunden und Arbeitszeitkonten. IAB-Bibliothek, Bd 361. Bielefeld: W. Bertelsmann Verlag. 